A us dem Anthropologibchen Institut der Universität Brestau.

(Direktor: Prof. Dr. Mollison.)

\title{
DER GEHIRNREICHTUM DER AUSTRALIER UND ANDERER HOMINIDEN, BEURTEILT NACH IHREM SKELET.
}

\author{
VON \\ OTTO HAUGER.
}

Mit 4 Abbildungen im Text.

Anntomigehe Hefte. I. Ahtoilung. 179. Heft (58. Bd., H. 3). 

Hoch über der Tierreihe, gemessen an ihren geistigen Fähig. keiten, steht der Mensch, äusserlich schon gekennzeichnet durch die mächtige Entfaltung seines Neurocraniums. Seit man erkannt hat, dass die psychischen Funktionen des Menschen sich auf der somatischen Grundlage seines Gehirns abspiclen, haben Naturforscher und Ärzte sich bemüht, einen Zusammenhang zwischen beiden zu finden. Aristoteles hat noch geglaubt, dass der Mensch unter allen Lebewesen das schwerste Gehirn habe. Man ist bald zur Erkenntnis gekommen, dass man mit absoluten Zahlen nichts anfangen kann, sondern dass man das Gehirngewicht in Beziehung zu den übrigen Abmessungen des Individuums setzen muss. Das Gehim ist ein Teil des Zentralnervensystems, die Grösse der Zentrale aber ist abhängig von der Grởsse und den Funktionen des Apparats, dem sie vorsteht. Man hat infolgedessen das Gehirngewicht in Vergleich gesetzt mit dem Körpergewicht und der Körperlänge. A. Brandt (1) hat zum ersten Male den Zusammenhang zwischen dem Hirngewicht und dem Körpergewicht innerhalb der Tierreihe näher untersucht und erkannt, dass die Zunahme des Gehirngewichts der Zunahme des Körpergewichts nicht direkt proportional ist, sondern dass die kleinen Tiere ein relativ grösseres Gehirn als die grossen haben. Er kam zu dem Schluss, dass es vor allem die somatischen Funktionen des Gehirns sind, die beim kleinen Individuum eine grössere Gehirnmasse verlangen. Th. L. W. v. B i s ch off (2) hat dann eine Reihe von Untersuchungen über das Hirngewicht des Menschen zusammengefasst. An einem grossen Material 
geht er all den Faktoren auf den Grund, die die Grösse des menschlichen Hirngewichts beeinflussen. Retzius (3), Marchand (4), Matiegka (5), Dräsek e (6), Hand mann (7), Wendt (8) und eine Anzahl anderer haben weiteres Material für die Gewichtsverhältnisse des menschlichen Gchirns gesammelt, und veröffentlicht. Die Ergebnisse der einzelnen Autoren lassen sich nicht ohne weiteres miteinander vergleichen, da ihre Technik der Wägung eine verschiedene ist. Immerhin aber lassen sich aus ihren Arbeiten die Faktoren deutlich ersehen, von denen beim Menschen und analog bei anderen Tieren die Grösse des Hirngewichts abhängig ist.

Es sind die folgenden:

1. Das Gehirn verändert sich mit dem Lebensalter. Es wächst in den ersten Lebensjahren sehr rasch, erreicht mit dem 20. Lebensjahr sein grösstes Gewicht, um schliesslich vom 50. Lebensjahr an auch seinerseits der senilen Involution zu verfallen.

2. Das Geschlecht. spielt. eine bedeutsame Rolle; das grössere absolute Hirngewicht des Mannes, das kleinere des Weibes sind bekannt. Das relative Gehirngewicht lässt die Frau allerdings besser dastehen.

3. Mit zunehmender Körpermasse, die sich ausdrücken lässt im Körpergewicht, wie auch mit zunehmender Körperlänge wächst das Hirngewicht.

4. Körperliche Fntwickelung und Ernährungszustand spielen eine Rolle.

5. Der Einfluss der Kultur auf die Gehirnmasse ist vie] umstritten. Broca an Pariser und E. Schmidt an Ägypterschädeln haben geglaubt, ihr eine günstige Einwirkung auf die Gehimentwickelung zuschreiben zu sollen. Neuere Intersuchungen geben dem Unrecht.

6. Klar liegt der deutliche Unterschied des Gehirngewichts bei den verschiedenen menschlichen Gruppen zulage. 
7. Die Intelligenz ist ebenfalls einer der Faktoren, die das Hirngewicht mitbestimmen.

8. Die Bluttülle des untersuchten Gehirns, pathologische Anomalien, vorausgegangene körperliche und Geisteskrankheiten und die Methodik der Wägung beeinflussen das Ergebnis von Gewichtsbestimmungen.

Das Verhältnis des Ciehirngewichts zum Körpergewicht ist die Grundlage tür alle übrigen Korrelationen. Die Tatsache, dass das Gehirn mit zunehmender Körpermásse nicht in demselben Masse zunimmt, hat schon Brandt (1) erkannt. Sie machte den Satz, dass das relative (iehirngewicht ohne weiteres die Intelligenz anzeige, hinfällig. Es galt zu ergründen, welche Gesetzmässigkeit in der Zunahme beider Gewichte, des Gehirn- und Körpergewichls, herrschte. B ischoff (2) wie Manouvrier (9) haben berechnet, wieviel Gramm Gehirn auf Kilogramm Körpergewicht und auf Centimeter Körperlänge kommen, wie Gehirn und Körper in ihren Gewichtseinheiten in Mittel zunehmen, aber eine Gesetzmässigkeit erkannten sie nicht. Da kamen O. snel] 1891 (11) und unabhängig von ihm D ubois 1897 (12, 13) zu der Erkenntnis, dass sich die gesetzmässige Zunahme beider Gewichte durch eine einfache Formel ausdrücken liess. Es ergab sich nämlich hei Untersuchungen innerhalb der Tierreihe, wenn man von Tieren, die auf gleicher geistiger stufe standen, grosse und kleine (z. B. Katze und Löwe) miteinander verglich, dass die (iehirngewichte zunahmen, wie die 0,56. Potenzen der Körpergewichte. O. Snell gals für das' wechselseitige Verhältnis die Formeln an, die sich auch bei Dubois in derselben Form finden :

$$
\begin{gathered}
e=k \cdot s^{r} \\
E: e=S^{r}: s^{r} \\
(S: s)^{r}=E: e \\
r=\frac{\log E-\log e}{\log S-\log s}
\end{gathered}
$$




$$
\mathrm{k}=\frac{\mathrm{E}}{\mathrm{S}^{\mathrm{r}}}=\frac{\mathrm{e}}{\mathrm{s}^{\mathrm{r}}},
$$

wobei e das Gehirngewicht, s das Körpergewicht des kleineren Tieres, E und S das Gehirn- und Körpergewicht des grösseren Tieres bedeuten und $\mathrm{k}$ ein weiter unten zu besprechender Faktor ist. Die als Exponent des Körpergewichts auftretende Grösse r ist in ihrer Deutung sehr schwierig. Lapique $(16,17,18,19)$ bezeichnet sie in seinen Untersuchungen als eine ,puissance étrange". Ihre Erklärung ist gleichbedeutend mit der Aufhellung der Tatsache, dass ein kleineres Tier ein relativ grösseres Gehirn braucht als ein gleich intelligentes grösseres. O. Snell bringt das mit dem Stoffwechset in Zusammenhang. Das kleinere Tier hat infolge seiner grösseren Körperoberfläche einen grösseren Stoffwechselumsatz, für diese grösseren somatischen Funktionen bedarf es grösșerer nervöser Zentren, und deshalb hat es ein relativ grösseres Gehirn als das grössere 'Tier der gleichen Gattung. Dubois (14) weist darauf hin, dass es die Entwickelung der Sinnesorgane ist, die beim kleineren lier einen relativ grösseren Raum in Anspruch nehmen als beim grösseren, und ihrerseits auch wieder die grössere Gehirnmasse des kleineren Tieres bedingen. Wie dem auch sei, jedenfalls kommen er sowohl, wie Lapique, wie auch Girard (20) immer wieder für die Grösse $r$ innerhalb der Wirbeltierreihe zu Werten, die um 0,56 schwanken, vorausgesetzt, dass man Arten gleicher Organisation, gleicher Körperform und gleicher lebenswèise miteinander vergleicht.

Damit ist nun die Abhängigkeit des Hirngewichts vom Körpergewicht in ihren Zusammenhüngen gegeben. Es ist aber klar, dass auch der Grad der geistigen Fahigkeiten eines Tieres von Einfluss auf das Gehirngewicht ist. Das veranlasst Snell sowohl wie D ubois, einen Faktor einzuführen, der der Ausdruck für die psychischen Qualitäten des betreffenden Individuums sein soll und dem sinell die Bezeichnung „psychi- 
scher Faktor" und Dubois die Bezeichnung "Cephalisationskoeffizient" gibt und ihn mit k bezeichnet. So ist die Formel $\mathrm{e}=\mathrm{k} \cdot \mathrm{s}^{\mathrm{r}}$ entstanden.

Will man mit ihr arbeiten, so hat man selbstverständlich alle übrigen Faktoren, die ausser Körpergewicht und Intelligenz das Gehirngewicht beeinflussen, sorgfältig auszuschalten; es sind dies die Punkte 1 und 2,4 bis 6 und 8 der obigen $\mathrm{Zu}$ sammenstellung. Dann aber kann man die Formel benützen und aus ihr auf die Intelligenz der untersuchten Individuen interessante Schlüsse ziehen. D u bo is $(12,13,14,15)$, L a pique $(16,17,18,19)$, Girard (20) haben innerhalbl der ganzen Vertebratenreihe die Gültigkeit des Gesetzes erprobt. Als Endergebnis dieser Untersuchungen kommt D u bo is 1914 (15) für die Grösse $\mathrm{r}$ zu dem Werte 0,5576, gültig beim Vergleich von Vertebratenarten gleicher Organisation, gleicher Körperform und Lebensweise. Er macht aber dabei die Einschränkung, dass dieser Korrelationsexponent $r$, an grossen und kleinen Individuen der gle ichen Art berechnet, nur 0,232 beträgt. $\mathrm{Zu}$ demselben Ergebnis ist auch $\mathrm{L}$ a pi q u e gekommen.

L apique hat darauf hingewiesen, dass die rechnerischen algebraischen Ergebnisse sich graphisch sehr übersichtlich darstellen lassen, wenn man auf einem Koordinatensystem nicht die Körper- und Gehirngewichte selbst, sondern deren Logarithmen abträgt. Das ist begreiflich, denn in der Formel $\mathrm{e}=\mathrm{k} . \mathrm{s}^{\mathrm{r}}$ spielt die Grösse $r$, da sie Exponent ist, die entscheidende Rolle. Sie wird in der logarithmischen Formel zum Faktor und in der graphischen Darstellung mit logarithmischem Masssystem erhält sie ihren Ausdruck in einer Geraden, die mil der Horizontalen den durch $\mathrm{r}$ gegebenen Winkel bildet. Es ist der Winkel a, dessen Tangente die Grösse $r$ ist. Auf dieser Geraden aber liegen dann die mittleren Gehirngewichte verschieden grosser Individuen derselben Art und Intelligenz, sie wird für diese Tiere zur Isoneuralen. Auf diese Weise hat 
Lapique in übersichtlicher form das feste Verhältnis zwischen Körper- und Gehirngewicht innerhalb der ganzen Wirbeltierreihe zur Darstellung gebracht (16, S. 251) und gezeigt, dass die Höhenlage der durch $r$ gegebenen Linie von der Intelligenzentwickelung der betreffenden Tierart abhängig ist.

Es liegt auf der Hand, dass es gerade für den Anthropologen schwer ist, frisches Leichenmaterial .... deun solches muss es sein, sollen die Werte miteinander verglichen werden - zu gewinnen. Man hat zwar oft versucht [Eyerich und Lo ow en feld (21) und viele andere (ci. Martin, 22, S. 648)], Schädelumfang und andere Masskriterien für die Gehirnausbildung heranzuziehen und sie mit Körperlänge und anderen Vergleichswerten in Beziehung zu setzen. Dass man nach solchen Methoden aher nur grobe Werte erhalten kann, und dass dem Untersucher dabei die feinen Creselzmässigkeiten, die snell and Dubois aufgedeckl haben, entgehen, ist verständlich.

Dem Anthropologen steht viellach nur Skeletmaterial und für die fossilen Hominiden überhaupt nur solches zur Verfügung. Lr ist genötigt, damit fürlieb zu nehmen. Durch diese Tatsache veranlasst, hat Mollis on (23) darauf hingewiesen, dass das S nell-D u bois sche Gesetz auch am Skeletmaterial seine Giltigkeit hehält. Das Gehirngewicht ersetzl er durch den in der Untersuchungstechnik viel stabileren Wert der Schädelkapazität, von ihm $\mathrm{K}$ oder $\mathrm{k}$ bezeichnet. Statt des Körpergewichts nimmt er das Knochenvolumen der langen Extremitätenknochen und nennt diese Grösse $V$ oder $v$, je nachdem es sich um das grössere oder kleinere Tier handelt. Der Cephalisationskoeffizient $\mathrm{k}$ von $\mathrm{D} u$ bois wird bei ihm zum Cerebralkoeffizienten c. Die Grösse $r$ bleibt bestehen. Dann lauten die Formeln:

$$
\begin{aligned}
\mathrm{K} & =\mathrm{c} \cdot \mathrm{V}^{\mathrm{r}} \\
\mathrm{K}: \mathrm{k} & =\mathrm{V}^{\mathrm{r}}: \mathrm{v}^{r}
\end{aligned}
$$




$$
\begin{gathered}
\log \mathrm{K}-\log \mathrm{k}=\mathrm{r}(\log \mathrm{V}-\log \mathrm{v}) \\
\mathrm{r}=\frac{\log \mathrm{K}-\mathrm{\operatorname {log } k}}{\log \mathrm{V}-\log \mathrm{v}} \\
\mathrm{c}=\frac{\mathrm{K}}{\mathrm{V}^{\mathrm{r}}}
\end{gathered}
$$

Auch dieses Verhalten lässt sich nach Lapique genan so graphisch darstellen (Fig. 1 und 3), nur nennt man die Isoneurale dann besser Isencephale. Mollison hat die Grösse $r$, die uns auch hier vor allem interessiert, für den Gorilla (6 Exemplare) berechnet und erhält dafür den Wert 0,5804, der fast genau mit dem D u bo is schen $r$ übereinstimmt.

Die Giltigkeit des Duboisschen Geselzes auch am so gewählten Skeletmaterial erscheint im ersten Augenblick merkwürdig. Sie ist es aber nicht. Moll is on ersetzt einmal das Gehirngewicht durch die Schädelkapazität; zwischen beiden besteht ja eine ziemlich feste Korrelation, die von Welcker (24) und Manouvrier (25) schon zahlenmässig festgelegt wurde. Statt des unsicheren Faktors des Körpergewichts nimm! Mollison das Knochenvolumen der 6 markantesten Extremitätenknochen, Humerus, Radius, Ulna, Femur, Tibia und Fibula, das heisst er setzt statt des Körpergewichts oder was, da man innerhalb ähnlicher Arten vergleicht, ja auch dasselbe ist, statt des Körpervolumens nur einen bestimmten Teil desselben, einen Teil, der viel zuverlässiger ist, als die je nach dem Ernährungszustande stark schwankende Grösse des Körpergewichtes. Es bestehen also (natürlich nicht his in viele Dezimalen hinein mathematisch genall genommen) die festen Beziehungen :

Schädelkapazität $=$ Gehirngewicht $\cdot a=$ Gehirnvolunen $\cdot a_{1}$;

Knochenvolumen $=$ Körpergewicht: $b=$ Körpervolumen $: b_{1}$. Daraus ergibt sich aber, dass man nach der Mollis on schen Methode zu denselben Werten für $r$ gelangen muss wie nach 
der Dubois schen, denn es ist irrelevant, ob man in der Formel

$$
\mathrm{r}=\frac{\log \mathrm{K}-\log \mathrm{k}}{\log \mathrm{V}-\log \mathrm{v}}
$$

$\mathrm{K}$ und $\mathrm{k}, \mathrm{V}$ und $\mathrm{v}$ nimmt, oder konstante Bruchteile dieser Grössen. Die. Differenzen der betreffenden Logarithmen sind dieselben. Die für den $\mathrm{D}$ u bo i s schen Koeffizienten der Cephalisation und den Mollisonschen Cerebralkoeffizienten gewonnenen Werte sind natürlich bei den beiden Methoden verschieden und miteinander nicht vergleichbar.

In einem aber, und das ist in der Genauigkeit, gebührt der Mollis on schen Methode vor der Dubois schen bei weitem der Vorzug. Für die Gehirngewichte erhält man so verschiedene Werte, je nach der Technik, nach der man arbeitet, je nachdem man die Medulla etwas weiter oben oder unten abschneidet usw., je nach dem Zustande des Gehirns, ob frisch oder konserviert. B i s ch off (2, S. 76) hat gezeigt, wieviel das Gehirn durch Konservierung mit der Zeit an Gewicht verlieren kinn. Blutfülle und vieles andere spielt noch eine Rolle. Demgegenüber hat unan in der Schädelkapazität, wenn man beim Menscher nur die Voraussetzung macht, sie erst bei Individnen vom 20. Lebensjahr an zu messen, etwas absolutes Festes, Starrwandiges in der Hand, das die Schrumpfung des Gehirns im Alter nicht mitmacht und das eine Konstante bleibt, ob ich sie jetzt oder erst nach vielen Jahren messe. Das Körpergewicht ferner ist derartig abhängig vom Ernährungszustand, von Veranlagung, von oft schwer fassbaren Krankheitszuständen und so vielem anderen, bei Haustieren von Zuchtergebnissen, von Domestikation. Wie will ich sagen, das und das Tier, der und der Mensch war in einem normalen Gewichtszustand? Das bleibt für das Tier noch mehr als für den Menschen, wo wir viele Vergleichszahlen haben, Schätzung. Leuret (26, S. 384) hat: schon, um einen krassen Fall herauszunehmen, ge- 
zeigt, dass sich das relative Gehirngewicht bei einer Gans, je nachdem sie gemästet ist, zwischen 1:467 und 1:3600 bewegt. Wenn ich aber das Körpergewicht zum Hirngewicht in der Snell-Dubois schen Formel in Beziehung setze, so the ich es deshallb, weil die nervöse Masse von der Körpermasse und weil die Grösse der Nervenzentrale von dem Umfange und der Zahl der Aussenstationen abhängt. Ich will wissen, wie ist denn das Verhältnis des Gehirns zu der Summe der innervierten Körperapparate? Da spielt das Fett nur eine untergeordnete Rolle, denn seine Innervation ist gleich Null. Da ist das Integument sowohl mit seinen Sinnesorganen wie vor allem der Lokomotionsapparat mit seinem Skeletsystem, seinen Muskeln und Bändern von entscheidender Wichtigkeit. So kann Mollison sehr wohl als Teil von ausschlaggebender Bedeutung das Skelet der Extremitäten beim Menschen und hochentwickelten Vertebraten in der Formel in Rechnung setzen. Und er wird, obwohl auch das noch nicht das Ideal ist, dabei genauere Werte erhalten, als nach der D u bo is schen Methode. Eine Einschränkung ist dabei zu machen: Das Extremitätenskelet kann nur bei den Tierarten zum Vergleich herangezogen werden, in deren Dasein die Extremitäten eine entscheidende Rolle spielen. Das ist der mit seinen Gliedmassen arbeitende Mensch, das sind die Affen, das sind die Tiere, für die die Schnelligkeit der Beine eine lebenswichtige Rolle spielt. Das sind vielleicht noch die Vögel, das ist wahrscheinlich nicht mehr das Hausschwein, das kann eine Schlange überhaupt nicht mehr sein.

Molli s on hat in seiner oben erwähnten Arbeit (23) den Gehirnreichtum der Primaten auf Grund ihres Extremitätenskelets nach der Snell-Duboisschen Formel und anderen Erwägungen beurteilt. Auf dieser Arbeit aufbauend und seine Ergebnisse heranziehend habe ich innerhalb der Species Mensch die Giltigkeit des Snell-Duboisschen Gesetzes und seine 
Folgerungen auf die (iehirnentwickelung und fulelligenz zu ergründen versucht.

Für meine Untersuchungen war ich auf das Material des Anthropologischen und des Analomischen Instituts der Universität Breslau angewiesen; Herr Prof. Mollis on und Herr Geh. Rat Kallius hatlen die Güte, mir es für meine Untersuchungen zur Verlügung zu stellen. Herr Prof. Mollison gab mir die Anregung zu dieser Arbeil, er stand mir auch während derselben stels gerne mit Ral und Tat zur Seile. Ich bin ihm dafür zu grösstem Danke verpflichtet.

Bei unserem geringen Besitzstande an eindeutigen und kompletten Rasseskeleten war eine grössere Serie von Australierskeleten, die Kla a $\mathrm{sch}$ von seiner australischen Reise mitbrachte (27), besonders wertvoll. 20 liessen sich für meine Intersuchungen verwerten. Sie stammten mit zwei Ausnahmen alle von der Küste von Queensiand. Die skelete haben den grossen lorzug, einer homogenen Gruppe anzugehören und rühren von Individuen her, die an Körpergrösse und deshalh auch an Knochenvolumen erheblich voneinander verschieden waren. Das war zur Eruierung der Geselzmissigkeit irn Wachsturn der Grössen Schïdelkapazilät und Knochenvolumen sehr schätzenswert. Als eurupäische Vergleichsgruppe konnte ich 11 Europiterskelete der analomischen Sammlung Breslau heranziehen. Ihre Herkunft ist leider nicht so eindeutig, wie die der Australier, sie stammen wohl meist aus dem Osten Deutschlands, wornit, rassenmäissig gesprochen, allerdings nichls gesagt ist. Diese Europäergruppe, deren Individuenzahl auch etwas klein isl, kann also lange nicht so wie die Australiergruppe als etwas Homogenes aulgefasst.werden, eine grössere Streuung in ihren Werten war voll vornherein zu erwarten. Sechs Negritos entnahn ich der Arbeit Mollisons. Der Rest der Individuen ist aus der labelle f ersichtlich. Es ist selbstverständlich, dass nur solche Skelete zur Untersuchung 
Der Gehirnreichtum der Australier und anderer Hominiden etc. $\quad 589$

Tabelle $I$.

\begin{tabular}{|c|c|c|c|c|c|c|c|c|c|c|}
\hline & & & 妾 & 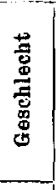 & 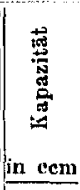 & 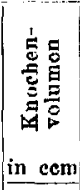 & $\begin{array}{l}\text { Rola- } \\
\text { tive } \\
\text { Kapa- } \\
\text { zität } \\
=\frac{K \cdot 100}{V}\end{array}$ & $\begin{array}{c}\text { Soma- } \\
\text { tisehe } \\
\text { Kompo- } \\
\text { neerte } \\
\text { V. } 39 \\
=\frac{100}{100} \\
\text { in cem } \\
\end{array}$ & $\begin{array}{l}\text { Poycli- } \\
\text { sche } \\
\text { Kompo } \\
\text { nente } \\
=\mathrm{K}-\mathrm{K} \\
\text { Som.- } \\
\text { Konip. } \\
\text { in cem } \\
\end{array}$ & $\begin{array}{c}\begin{array}{c}\text { Crehral- } \\
\text { ooefa- } \\
\text { zient } \\
=\frac{K}{V^{0,25}}\end{array} \\
\end{array}$ \\
\hline 1 & & 2 & 3 & 4 & 5 & 6 & 7 & 8 & 9 & 10 \\
\hline \multirow[t]{20}{*}{ A ustralier } & \multirow[t]{20}{*}{$\mathrm{Br}$} & 1004 & 1 & t & 1038 & 597 & 174 & 233 & 805 & 210,0 \\
\hline & & 1006 & 2 & $\sigma^{2}$ & 1168 & 955 & 122 & 373 & 795 & 210,1 \\
\hline & & 1007 & 3 & Q? & 1220 & 760 & 160 & 296 & 924 & 237,9 \\
\hline & & 1008 & 4 & $\sigma^{\pi}$ & 1185 & 869 & 137 & 339 & 746 & 218.2 \\
\hline & & 1009 & 5 & 0 & 1235 & 1312 & 94 & 518 & 717 & 204,8 \\
\hline & & 1010 & 6 & $\sigma^{2}$ & 1252 & 867 & 145 & 339 & 913 & 230,7 \\
\hline & & 1016 & 7 & $q$ & 1130 & 595 & 190 & 233 & 897 & 229,2 \\
\hline & & 1017 & 8 & ㅇ? & 1153 & 745 & 155 & 292 & 861 & 220,8 \\
\hline & & 1020 & 9 & 0 & 1198 & 605 & 197 & 236 & 957 & 240,5 \\
\hline & & 1021 & 10 & q & 965 & 379 & 255 & 148 & 817 & 218,6 \\
\hline & & 1028 & 11 & む? & 1056 & 476 & 222 & 186 & 870 & 226,0 \\
\hline & & 1031 & 12 & q & 1093 & 496 & 221 & 194 & 899 & 231,6 \\
\hline & & 1033 & 13 & 0 & 1141 & 722 & 158 & 282 & 859 & 220,0 \\
\hline & & 1034 & 14 & $\sigma^{3}$ & 1233 & 1020 & 121 & 398 & 835 & 218,1 \\
\hline & & 1039 & 15 & q & 928 & 573 & 162 & 224 & 704 & 189,7 \\
\hline & & 1042 & 16 & 0 & 1341 & 880 & 153 & 344 & 997 & 246,8 \\
\hline & & 1043 & 17 & 9 & 1240 & 664 & 187 & 259 & 981 & 244,3 \\
\hline & & 1044 & 18 & 9 & 1089 & 714 & 153 & 279 & 810 & 211,1 \\
\hline & & 1070 & 19 & $\sigma^{7}$ & 1225 & 991 & 124 & 387 & 838 & 218,3 \\
\hline & & 1073 & 20 & q & 1041 & 634 & 164 & 247 & 794 & 207,5 \\
\hline \multirow{11}{*}{ Europiler } & NC & 122 & 21 & $\sigma^{7}$ & 1704 & 1267 & 135 & 495 & 1209 & 285,6 \\
\hline & $\mathrm{NC}$ & 128 & 22 & $\sigma^{3}$ & 1254 & 1062 & 118 & 414 & 840 & 219,7 \\
\hline & \multirow[t]{8}{*}{$\mathrm{NC}$} & 364 & 23 & $\sigma^{i}$ & 1504 & 1125 & 134 & 438 & 1066 & 259,7 \\
\hline & & 367 & 24 & $\sigma^{7}$ & 1391 & 1241 & 112 & 484 & 907 & 234,3 \\
\hline & & 368 & 25 & $0^{7}$ & 1646 & 1022 & 161 & 399 & 1247 & 291,1 \\
\hline & & 369 & 26 & б. & 1381 & 1089 & 127 & 426 & 955 & 240,4 \\
\hline & & 370 & 27 & 8 & 1426 & 1111 & 128 & 434 & 992 & 247,0 \\
\hline & & 696 & 28 & $0^{7}$ & 1402 & 1058 & 132 & 413 & 989 & 245,9 \\
\hline & & 697 & 29 & $0^{x}$ & 1993 & 1250 & 103 & 488 & 805 & 217,5 \\
\hline & & 698 & 30 & $\sigma^{7}$ & 1658 & 1066 & 156 & 416 & 1242 & 286,2 \\
\hline & $\mathrm{AH}$ & 25 & 31 & q & 1368 & 831 & 165 & 324 & 1044 & 254,7 \\
\hline \multirow{7}{*}{$\begin{array}{c}\text { Negrito oder } \\
\text { Aëta }\end{array}$} & & & & & & & & & & \\
\hline & St & $3828 *$ & 32 & $0^{x}$ & 1266 & 579 & 218 & 226 & 1040 & 258,0 \\
\hline & & $2626 *$ & 33 & $q$ & 1100 & 497 & 221 & 194 & 906 & 298,5 \\
\hline & & $3828 a^{*}$ & 34 & $0^{7}$ & 1460 & 716 & 204 & 279 & 1181 & 282,2 \\
\hline & & $3828 b *$ & 35 & $\sigma^{x}$ & 1226 & 641 & 191 & 251 & 975 & 243,6 \\
\hline & & $2625 *$ & 36 & $\sigma^{7}$ & 1246 & 551 & 226 & 215 & 1081 & 257,2 \\
\hline & & $3828 \mathrm{c}^{*}$ & 37 & $0^{7} ?$ & 1522 & 566 & 269 & 221 & 1301 & 312,0 \\
\hline
\end{tabular}




\begin{tabular}{|c|c|c|c|c|c|c|c|c|c|c|}
\hline & & & 嵌 & 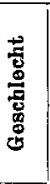 & 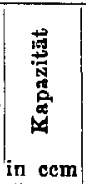 & 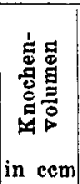 & $\begin{array}{c}\text { Rela- } \\
\text { tive } \\
\text { Kapi- } \\
\text { zitat } \\
\text { K.100 } \\
=\frac{V}{V}\end{array}$ & $\begin{array}{c}\text { Soma- } \\
\text { tisehe } \\
\text { Konpo- } \\
\text { nentte } \\
=\frac{\mathrm{v} .39}{100} \\
\text { in } \mathrm{ccm}\end{array}$ & \begin{tabular}{|c|} 
Pogchi- \\
sche \\
Kompo- \\
nente \\
$=\mathrm{K}-$ \\
Som- \\
$\mathbf{K o m p}$. \\
in ccm
\end{tabular} & $\begin{array}{l}\text { Cerehral- } \\
\text { koeffi- } \\
\text { zient } \\
=\frac{K}{\mathbf{v}^{0,2 S}}\end{array}$ \\
\hline 1 & & 2 & 3 & 4 & 5 & 6 & 7 & 8 & 9 & 10 \\
\hline \multirow[t]{2}{*}{ Lappländer } & & 12 & 38 & † & 1283 & 617 & 208 & 241 & 1042 & 257,4 \\
\hline & N & 12 & 9 & 8 & 1490 & 941 & 158 & 367 & 1123 & 269,0 \\
\hline \multirow[t]{2}{*}{ Igorote. . } & $\mathrm{NC}$ & 125 & 40 & $\sigma^{2} ?$ & 1151 & 590 & 195 & 231 & 920 & 233,6 \\
\hline & $\mathrm{NC}$ & 127 & 1 & $\sigma^{\pi}$ & 1249 & 868 & 144 & 338 & 911 & 230,2 \\
\hline stin & $\mathrm{NC}$ & 23 & 42 & $\sigma^{x}$ & 1212 & 893 & 136 & 349 & 863 & 221,7 \\
\hline ann $\cdot$ & $\mathrm{NC}$ & & 10 & $8 ?$ & 1260 & 891 & 141 & 349 & 911 & 236,0 \\
\hline ara & St & $3982 *$ & 44 & $\sigma^{\pi}$ & 1573 & 730 & 215 & 285 & 1288 & 302,7 \\
\hline & $\mathrm{AH}$ & $8 \Delta$ & 45 & $\sigma^{\prime}$ & 1338 & 1126 & 118 & 489 & 894 & 230,1 \\
\hline eger. . . & $A H$ & $158^{*}$ & 46 & $\sigma^{7}$ & 1388 & 947 & 147 & 369 & 1019 & 250,8 \\
\hline leander & & & 47 & & $12: 30$ & 1106 & 111 & 433 & 797 & 212,2 \\
\hline
\end{tabular}
nommen.

Die mit * bezeichneten Individuen sind der Mollis o n schen Arbeit ent-

herangezogen wurden, bei denen alle Knochen zweifelsfrei von demselben Individuum stammten. Das ist bei vielen Sammlungsskeleten nichl ohne weiteres der Fall.

Von sämtlichen Skeleten wurde die Schädelkapazität und das Volumen der langen Röhrenknochen (Humerus, Radius, Ulna; Femur, Tibia und Fibula) der rechten Körperhälfte, und, wenn sie fehlten, der linken Seite nach der von Mollis on (23, S. 389) angegebenen Methode mit Rübsamen und unter Benützung der dort beschriebenen Messinstrumente gemessen. Die gefundenen Summenwerte für die Einzelindividuen sind in der Tabelle I niedergelegt. Die Ergebnisse wurden in der Fig. 3 nach dem Lapique schen Veríahren, indem auf der Abszisse der Logarithmus des Knochenvolumens und auf der Ordinate der Logarithmus der Schädelkapazität abgetragen wurde, übersichtlich dargestellt. 
Dabei ergab sich nun, dass die von $\mathrm{D} u$ bo o i s für die ganze Wirbeltierreihe als giltig stabilisierte Formel $\mathrm{E}=\mathrm{k} . \mathrm{S}^{0,58}$ und die von Mollison für die Anthromorphen am Knochenmaterial errechnete Formel $\mathrm{K}=\mathrm{c} \cdot \mathrm{V}^{0.58}$ nicht mehr für den Menschen gilt. Denn für den Menschen nimmt der Exponent $r$ einen anderen Wert an, er wird kleiner. Schon Mollis on hat erkannt, dass innerhalb der Primaten die niederen Ostaffen das grösste, die höheren Affen ein kleineres und die Menschen das kleinste $\mathrm{r}$ besitzen. Sein geringes Material gestattete ihm nicht, zu genauen Ergebnissen für den Menschen zu kommen.

Der Wert der Grösse r ergab sich aus meinen Zahlen für den Menschen als $\mathrm{r}=0,25$, ich errechnete ihn für die höheren Affen nach Mollison als $r=0,43^{1}$ ) und für die niederen Ostaffen als $\mathrm{r}=0,60$. In der graphischen Darstellung (Fig. 1) ist diese Veränderung von $r$ innerhalb der Primaten deutlich zu erkennen, denn die Grösse $r$ findet in der Neigung der Isencephalen ihren klaren Ausdruck, da sie ja die Tangensfunktion des Winkels ist, den diese Linie mit, der Horizontalen bildet.

Lapique (17) und auch D ubois in seiner letzten Arbeit (15) finden nun ebenfalls für den Menschen innerhalb seiner Art den Wert für $r=0,25$. La pique fand ihn aber auch für den Haushund giltig (17, S. 314) und J) u bo is (15, S. 336) auch lïr Eichhörnchen und Ochsenfrösche ${ }^{2}$ ). Der domestizierte Haushund ist ein ungünstiges Studienobjekt, das zugrunde liegende Material Dubois' ist, da es sich immer nur um sehr

1) Ich komme hier zu einem anderen Wert făr $r$ für die höberen Affen als Mollison, weil Mollison diesen Wert nur aus den 6 Gorillas berechnete, ich aber aus allen 12 Anthropomorphen der Mollisonschen Veroffentlichung.

') Die Ergebnisae Lapiques am Haushund wurden neuerdinge bestătigt durch B. Klatt (zur Methodik vergleichender metrischer Untersuchungen, besonders des Herzgewichts. Biol. Zentralbl. Bd. 39. 1919. S. 413). Auch or weist dabei auf die Domestikation hin. 
wenige Individuen handelt, anfechtbar. Nur für den Menschen verfügen beide über hinreichende und gute Grundlagen. D u bo is hat, von diesen Ergebnissen aus den Untersuchungen innerhalb verschiedener kleiner Wirbeltierarten geleitet, eine neue Gesetzmässigkeit abgeleitet, indem er sagt: Die Gehirn-

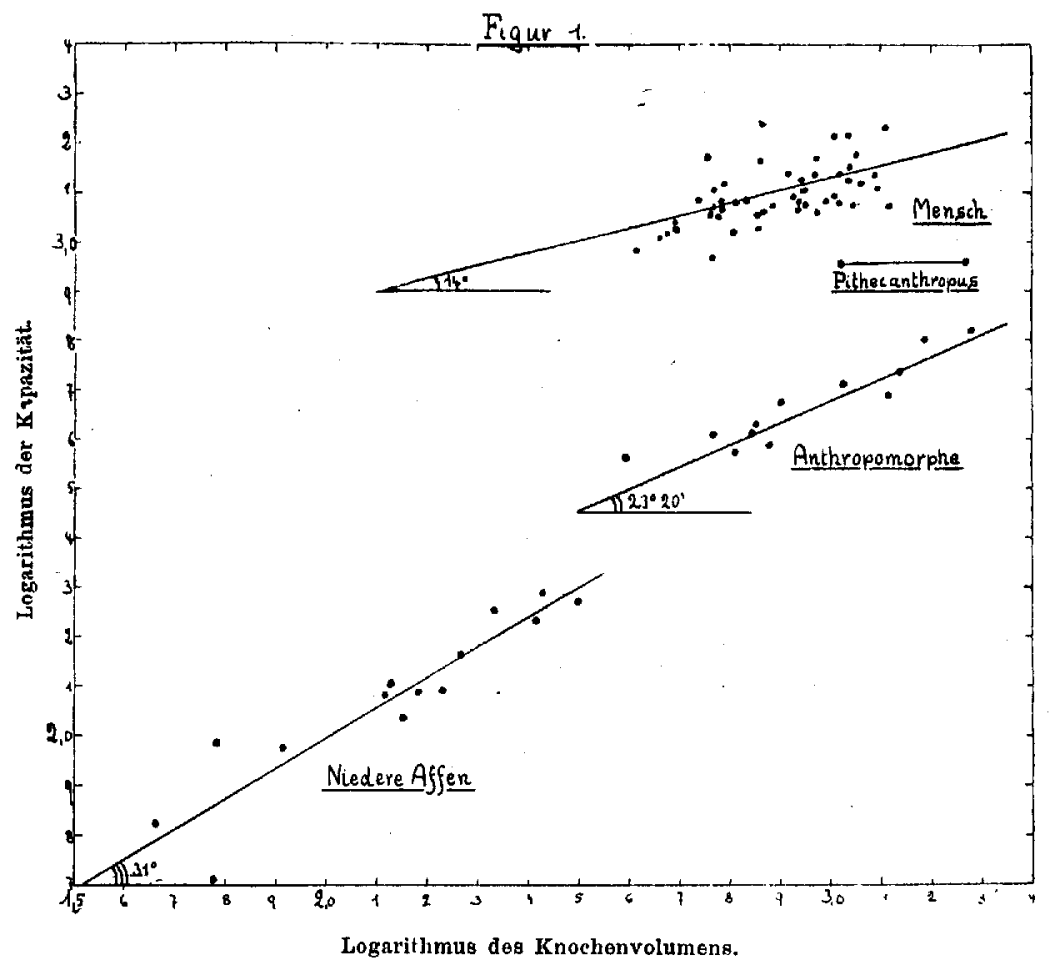

Kapazität und Knochenvolumen im logarithmischen Koordinatensystem.

Horizontal liegt der logarithmische Massstab des Extremitätenknochenvolumens, vertikal der logarithmische Massstab der Kapazität. Die der Horizontalen zugeneigten Geraden sind die Isencephalen.

gewichte verhalten sich bei Individuen innerhalb einer Art wie die 0,22. Potenzen der Körpergewichte, während sonst für Individuen von Vertebratenarten gleicher Organisation, Körperform und Lebensweise die 0,56. Potenzen in Frage kommen. 
Er hat versucht, diese Merkwürdigkeit auch zu erklären. Sein Gesetz innerhalb der Art steht auf schwachen Füssen, frappierend aber ist, dass sich aus meinen Berechnungen dieselbe Grösse $\mathbf{r}=0,25$ für den Menschen ergibt, wie sie Lapique und Dubois mit ganz anderen Methoden gefunden haben.

Die Berechnung der Grösse $r$ erfolgte aus folgenden Erwägungen heraus. Die Einzelkapazitäten und Einzelvolumina sind Gruppen von zwei Merkmalen, die in Beziehung zueinander gesetzt werden sollen. Dass eine Gesetzmässigkeit in ihrem gegenseitigen Verhalten besteht, ist erwiesen. Die Korrelation zweier Merkmale lässt sich auf mathematischem Wege zum Ausdruck bringen, indem man den Korrelationskoeffizienten berechnet (Martin, 22, S. $78 \mathrm{f}$.). Im Koordinatensystem mit idem Massstab des einen Merkmals auf der Ordinate, dem Massstab des anderen Merkmals auf der Abszisse stellt sich dieser Korrelationskoeffizient $\mathbf{x}$ als eine Gerade dar, die mit der Horizontalen einen bestimmten durch $\mathbf{x}$ gegebenen Winkel bildet. Für je 2 Punkte auf dieser Geraden gilt dann das Gesetz:

$$
\mathrm{x}=\frac{\mathrm{K}-\mathrm{k}}{\mathrm{V}-\mathrm{v}} \text {. }
$$

Nun haben Snell, Dubois und Lapique aber erwiesen, dass sich die Gehirngewichte bzw. die Kapazitäten nicht wie die r-fachen Produkte der Körpergewichte bzw. der Knochenvolumina verhalten, sondern wie die r-ten Potenzen. Mit anderen Worten, sie sind $\mathrm{zu}$ keinem Korrelations k o f f $\mathrm{i}$ zienten gekommen, sondern zu einem Korrelations expon en ten. Der Korrelations ex p on en t.zweier Grössen ist aber mathematisch gleich dem Korrelationskoeffizienten der L o garithmen dieser beiden Grössen. Ich brauche also nur den Korrelationskoeffizienten für die Logarithmen der schädelkapazitäten und die Logarithmen der Knochenvolumina zu berechner und ich habe die Korrelation zwischen Kapazität und 
Volumen, wie sie die Snell-D uboissche Formel verlangt. Dieser neue Korrelationskoeffizient $\mathrm{r}$ hat dann für je 2 Individuen folgende Eigenschaft:

$$
r=\frac{\log K-\log \mathrm{k}}{\log \mathrm{V}-\log \mathrm{v}}
$$

Mit derselben Gleichung aber haben schon S nell and D a bo is (siehe oben) die Grösse r ausgedrückt. Das gefundene r hat ulann die Eigentümlichkeit, im Koordinatensystem, auf dem ich jetzt aber die Massstäbe der Logarithmen abtragen muss, in einer Geraden seinen Ausdruck zu finden. Dieses logarithmische Koordinatensystem ist aber nichts anderes, als die $L$ a piquesche Anordnung und die durch $r$ gegebene Gerade ist die L a p i q u e sche lsoneurale und die Molli s on sche lsencephale.

Nun lässt sich der Korrelationsexponent für eine ganze Gruppe nach der folgendermassen modifizicrten Pearsonschen Formel (cf. Martin, 22, S. 79) berechnen:

$$
r=\frac{\boldsymbol{s}\left(\log \mathrm{K}-\log \mathrm{M}_{\mathrm{K}}\right)\left(\log \mathrm{V}-\log \mathrm{M}_{\mathrm{v}}\right)}{\mathbf{n} \cdot \sigma_{\log K} \cdot \sigma_{\log } \mathrm{v}}
$$

$M_{K}$ und $M_{v}$ sind die Mittelwerte für Kapazität und Knochen volumen innerhalb der untersuchten Gruppe, $\mathrm{n}$ die Individuenzahl, $\sigma_{\log \mathrm{K}}$ und $\sigma_{\log \mathrm{v}}$ die stetigen Abweichungen der Logarithmen beider Merkmale.

Diese Methode, $\mathbf{r} z u$ berechnen, ist zwar umständlich, aber genau und mathematisch einwandfrei. Nach ihr berechnet ist für den. Australier $r=0,2279$ und der wahrscheinliche Fehlel $\mathrm{E}_{\mathrm{r}}=0,1428$.

Man kommt auf wesentlich einfacherem Wege zu denselben Resultaten für $r$ mit etwas ver m inderter Genauigkeit, wenn man folgendermassen vorgeht: Man teilt die zu untersuchende Gruppe durch den Mittelwert ihrer Einzelvolumina in zwei Hälften, in eine, der die Individuen mit grösseren Voluminis als der Mittelwert angehören, und eine andere Hälfte, in der sich alle anderen Individuen mit kleineren Voluminis befinden. 
Der Gehirnrejchtum der Australier und anderer Hominiden etc. 595

Für beide Hälften gesondert werden nun die Mittelwerte für $\mathrm{V}$ und $\mathrm{K}$ berechnet, und aus diesen Mittelwerten ergibt sich dann $\mathbf{r}$ nach der Formel

$$
r=\frac{\log K-\log k}{\log V-\log v}
$$

Zeichnel man die gefundenen beiden Mittelwerte für beide Hälften in der Lapique schen Anordnung (Fig. 1 und 3) ein, so braucht man beide Punkte nur zu verbinden und man ist auch auf graphischem Wege zu $\mathbf{r}$ gekommen. Dieser kürzere Weg der Berechnung wurde in dieser Arbeit eingeschlagen, nach ihm ist z. B. für den Australier $r=0,253$.

Was sagt uns nun der verschiedene Wert für die Grösse $r$ innerhalb der Primatenreihe aus, was bedeutet das, dass er für den Menschen kleiner ist als für die Affen und übrigen Wirbeltiere? Das lässt uns das Snellsche Gesetz erkennen, denn in der Formel $K=c . V^{r}$ haben wir noch einen Koeffizienten c, der eingeführt ist, um ceteris paribus die Intelligenz zu beurteilen:

Je weniger bei der Zunahme des Knochenvolumens die Schädelkapazität gesetzmässig innerhalb einer Art steigt, desto geringer ist die Grösse $r$, desto geringer ist die Neigung der Isencephalen, desto grösser wird aber der Cerebralkoeffizient, das heisst, desto intelligenter ist die Art. Diejenige $\Lambda$ rt, bei der $r=1$ wäre, der Winkel der Isencephalen zur Horizontalen also $=45^{\circ}$, bei der also mit zunehmendem Knochenvolumen die Schädelkapazität in gleichem Verhältnis steigen würde, hätte den kleinsten Cerebralkoeffizienten, wäre an psychischen Qualitäten am ärmsten. Das würde heissen: Gleichmässig mit der Körpervergrösserung nimmt auch der dazu notwendige nervöse Apparat an Grösse zu, ohne dass etwas von nervösen Elementen vorhanden, wäre, was über diese somatischen Anforderungen hinaus eine psychische Rolle spielte. Der Cerebralkoeffizient 
wäre dann einfach $=\frac{K}{V}$, das heisst, er wär'e eine Grösse um 1 oder ein echter Bruch ${ }^{1}$ ), während er beim Affen etwa 10 und beim Menschen um die 250 beträgt. Etwas Derartiges gibt es aber praktisch schon deshalb nicht, weil ja nachgewiesen ist, dass kleinere Tiere ein relativ grösseres Gehirn haben, als grössere: Es ist anzunehmen, dass der grösste Wert, den $r$ in der Wirbeltierreihe annehmen kann, eben jener Zahl $\mathrm{r}=0,56$ nahesteht, die in allen Arbeiten wiederkehrt.

Je mehr aus dem Centralnervensystem, soweit wir es messbar in der Schädelkapazität in der Hand haben, und das ursprünglich nur den Organismus reguliert, je mehr aus ihm eine Psyche herauswächst, sich höhere über die Bedienung des körperlichen Apparats hinausgehende geistige Fähigkeiten entwickeln, desto geringer wird die Neigung der Isencephalen zur Horizontalen, desto kleiner wird der Wert für $r$. Der psychische Anteil des Gehirns spielt dann eine solche Rolle, dass er die gesetzmässige Zunahme der für die Körperfunktionen notwendigen Gehirnmasse bei zunehmender Körpermasse verdeckt.

Würde der Wert von $\mathbf{r}=0$ sein, so wäre der Cerebralkoeffizient $\mathbf{c}=\frac{\mathrm{K}}{\mathrm{V}^{\mathrm{v}}}$, das heisst, er wäre die Schädelkapazität selber, es wäre alles nur Psyche, somatische Funktionen wären überhaupt nicht mehr vorhanden. Etwas derartiges nur Psychisches und Körperinnervationsloses gibt es nicht.

Was mit diesen Sätzen ausgesprochen ist, ist eine Vermutung, die sich aus dem Verhalten der Grösse $r$ innerhalb der Affen- und Menschenreihe ableiten lässt, und die an Wahrscheinlichkeit beim Durchdenken der zahlenmässigen Untersuchungsergebnisse gewinnt. Es ergibt sich daraus, dass die Dinge

1) Innerhalb der Stugetierreihe gibt es kaum Individuen, deren Schadel. kapazität viel grösser ist, als das Volumen ihrer 6 Extremitätenknochen. 
folgendermassen liegen: Der den somatischen Funktionen (lienende Gehirnanteil nimmt mit zunehmender Körpermasse zu, und zwar stärker als die 0,58. Potenzen der Körpervolumina bzw. der Knochenvolumina. Der die Grundlage der Psyche bildende Himanteil ist von dem Körpervolumen vermutlich ebenfalls nicht unabhängig, sondern nimml wohl auch zu, aber nur sehr minimal. Bei den niederen Primaten, wo die psychische Komponente des Gehirns noch schlecht entwickelt ist, entsteht aus der Zunahme beider Gehirnteile eine Resultierende, die $r=0,60$ ist. Beim Menschen, wo der stark entwickelte psychische Hirnteil sehr äberwiegt, wird dadurch $\mathrm{r}$ zum Wert 0,25 herabgedrückt.

Es ist nun wohl möglich zu sagen, wo die rein somatischen und wo gewisse psychische Funktionen des Individuums in seinem Gehim ungefähr ihren Sitz haben (Flechsig, 28). Man kann aber die beiden Massenteile des Gehirns, die Psyche und Soma dienen, nicht gewichlsmässig voneinander trennen. Infólgedessen können wir auch die Grössen $r$ für die somalische und psychische Komponente des Gehirns nicht einzeln bestimmen, sondern müssen uns mit obiger Überlegung bescheiden.

Wohl aber können wir an dieselbe Frage noch von einer anderen Seite herantreten.

Wir lassen dabei das Snell-D uboissche Gesetz ganz beiseite. Dubois hat der Vereinfachung halber angenommen, dass somatischer wie psychischer Hirnanteil in gleichem Masse vom Körpergewicht abhängig sind. Wir sind eben darauf gekommen, dass das nicht richtig sein kann, und haben erkannt, dass Dubois, wenn er ein konstantes und nicht wie wir ein innerhalb der Wirbeltierreihe abnehmendes $\mathrm{r}$ annimmt, die psychische Komponente mit zunehmendem Körpervolumen zu stark wachsen lässt. Wir wollen nun ins, andere Extrem verfallen und von der Voraussetzung ausgehen, dass die psychische 
Komponente überhaupt nicht wachse, sondern absolut konstant sei, wie es auch Mollis on versuchsweise für die Anthropomorphen in seiner Arbeit angenommen hat (23, S. 391). Es erhebt sich dabei für uns die Frage: Welchen Prozentsatz des Knochenvolumens als Ausdruck der somatischen Komponente des Gehirns müssen wir von der Schädelkapazität abziehen, um einen stets konstant bleibenden Rest der Schädelkapazität, der die psychische Komponente sein soll; zu erhalten? Da sich feststellen lässt, welche Kapazität im Mittel jedem Knochenvolumen entsprichl, brauchen wir das nur an diesen Mittelwerten zu erproben. Man erhält für die psychische Komponente die konstantesten absoluten Werte, wenn man annimmt, dass die für rein körperliche Bedürfnisse notwendige Kapazität $39 \%$ des Volumens der Extremitätenknochen ausmache. Der konstante psychische Anteil der Kapazität beträgt dann, an diesen Mittelwerten berechnet, bei grossen wie kleinen Individuen ungefähr $955 \mathrm{ccm}$. Bei den Anthropomorphen ist Mollison, als er ihre psychische Komponente der Kapazität als konstant annahm, auf etwa 15\% des Extremitätenvolumens für den somatischen Anteil gekommen und auf einen für die höheren Funktionen übrigbleibenden Kapazitätsrest von $300 \mathrm{ccm}$.

Es ist aber nicht anzunehmen, dass der Mensch einen über doppelt so grossen Prozentsatz seines Gehirns zur Regulierung seiner körperlichen Bedürfnisse benötigen sollte, als der höhere Affe. Der Wert der somatischen Gehirnkomponente ist mit $39 \%$ zweifellos zu hoch. Es ist also nicht richtig, wenn man den psychischen Anteil als konstant und von der Körpergrösse unabhängig annimmt und nur den körperlichen wachsen lässt. Die somatische Komponente wird dann, da sie ja das ganze Wachstum allein darstellen soll, zu gross, sie nimmt sich noch ein Stück Psyche, wenn man so sagen darf, zu Hilfe. Denn sie kann ja gar nicht $39 \%$ betragen, sondern höchstens $15 \%$ wie beim Anthropomorphen, wahrscheinlich aber noch weniger, 
Der Gehirnreichtum der Australier und anderer Hominiden etc. 599

weil auch beim Anthropomorphen ein Fehler nach derselben Richtung vorliegt.

Man kommt so, von ganz anderen Voraussetzungen aus. gehend, zu demselben Schluss, dass nämlich die somatische Komponente des Gehirns mit zunehmender Körpermasse stark wachsen muss und die psychische Komponente keine Konstante ist, sondern ebenfalls wächst, wenn auch nur wenig.

Die logischen Schlüsse, die sich auf beiden Hypothesen als auf zwei Extremen aufbauen, nähern sich demselben einen Punkte, in dem die Wahrheit liegen muss.

Aber noch mehr sagt uns dieser neue Gedankengang. Wir haben als Mittelwert der psychischen Komponente innerhalb der ganzen von uns untersuchten Menschheitsgruppe ungeführ den Wert $955 \mathrm{ccm}$ erhalten und für die somatische Komponente die Grösse: 39\% des Knochenvolumens.

Wir brauchen nun nur bei allen Einzelindividuen 39\% ihres Knochenvolumens von ihrer Schädelkapazilät abzuziehen und haben dann die ihnen eigentümliche psychische Kompo. nente. Diese ist natürlich bei den Einzelindividuen nicht mehr gleich dem Mittelwert, sondern höher oder niedriger, je nach dem Masse der Cerebralisation des betreffenden Individuums, je nach dem Masse der Ausbildung seiner höheren Gehirnpunktionen.

Die psychische Komponente muss also dasselbe aussagen wie der Cerebralkoeffizient, da beide Werte ja letzten Endes auf denselben rechnerischen Grundlagen sich aufbauen. Die Grösse 39 in der Gleichung

$$
\text { Psychische Komponente }=K-\frac{V \cdot 39}{100}
$$

ist ja berechnet aus den sich entsprechenden Mittelwerten für $K$ und $V$, die nach der Formel

gefunden sind.

$$
c=\frac{\mathrm{K}}{\mathrm{V}^{0,25}}
$$


Das fast gleiche Verhalten von Cerebralkoeffizient und psychischer Komponente zeigt deutlich die Fig. 2. Sie gibt die Resultate der Tabelle I graphisch wieder. Auf der Horizontalen finden wir die laufenden Nummern der Einzelindividuen verzeichnet. Die untere Kurve ist aus den Einzelwerten der psychischen Komponente gewonnen; für sie gilt der linke Massstab. Die obere Kurve stellt das Verhalten des Cerebralkoeffizienten dar, ihre Werte- sind bezogen auf den

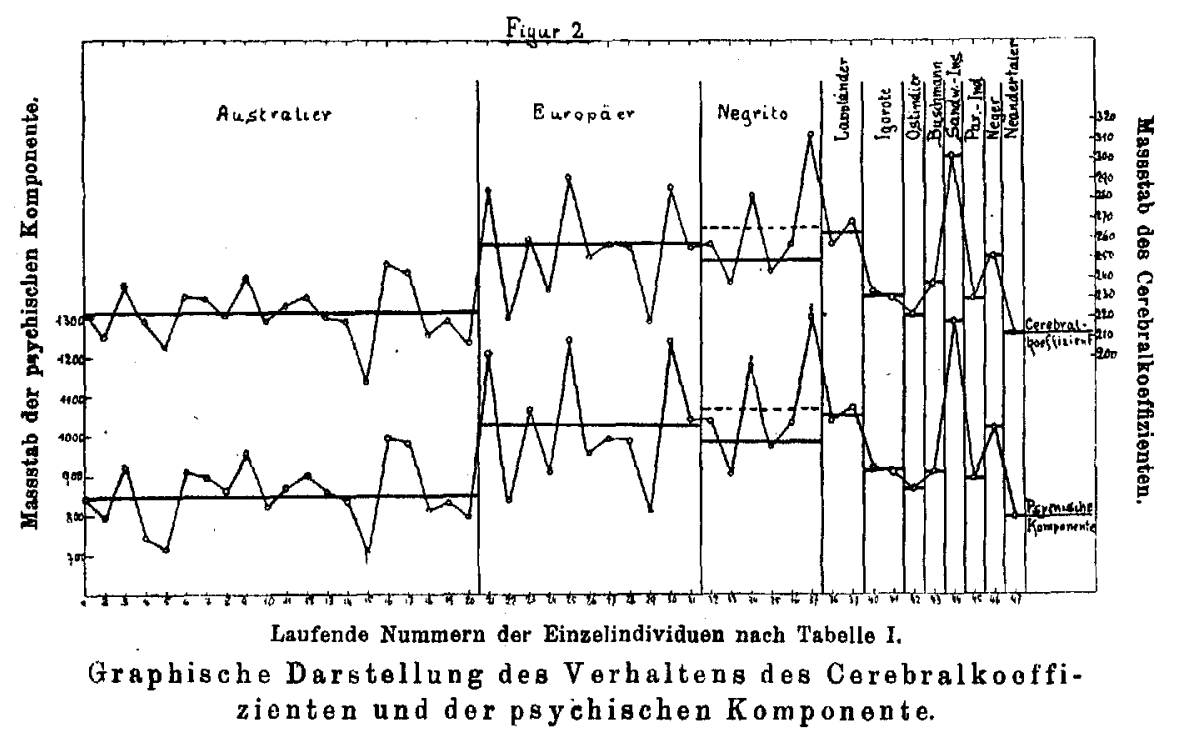

Die Horizontalen sind die Mittelwerte.

rechts verzeichneten Massstab. Wir erkennen in den Horizontalen die niederen Mittelwerte in psychischer Komponente und Cerebralkoeffizienten für den Australier, die höheren für den Europäer. (Gestrichelt ist die unter Einschluss der beiden traglichen Individuen berechnete Mittelwertslinie für die Negrito.) Wir sehen, dass die Kurve beim Australier nur schwachen, beim Europäer starken Schwankungen unterliegt, dass die Cerebralisation, die Entwickelung der Psyche inner- 
halb der Australiergruppe nicht so variabel ist wie beim Europäer.

Die graphische Darstellung der Fig. 1 führt unmittelbar in dic Fig. 3 ein. Die. Fig. 3 gibt in der L a pi qu e schen Anordnung die menschlichen Gruppen allein wieder.

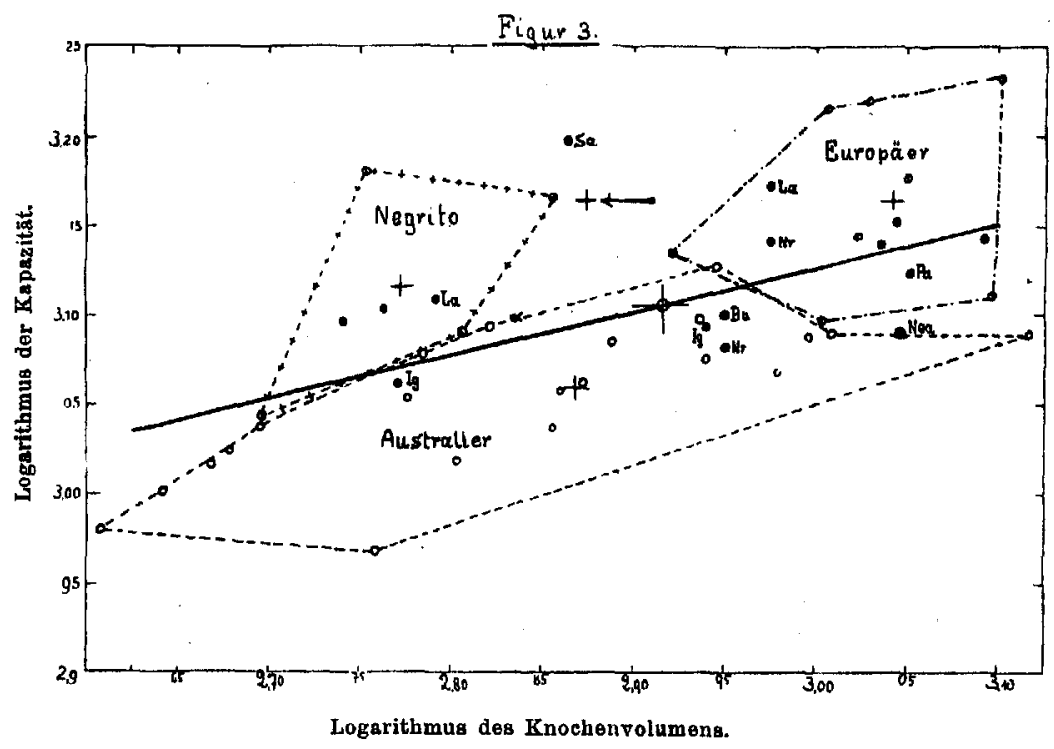

Kapazitat und Knoohenvolumen des Menschen, dargestellt im logarithmischen Koordinatensystem.

--- Australier, $-\frac{-}{\circ}$ Europker, $-+\frac{\odot}{\odot}+$ Negrito, - Skelete anderer Gruppen: Bu Buschmann, Ig Igorote, La Lappländer, Nea Neandertaler, Nr Neger und Ostindischer Neger, Pa Paraguay-lndianer, Sa Sandwich-Insu-

laner. + Mittelwerte der einzelnen Grappen, - Mittelwert Mensch,

$+\leftarrow$ Horizontalverschiebung des Mittelwertes der Europker (cf. S. 609).

Das Feld durchschneidet eine stark der Horizontalen zugeneigte Gerade; sie ist die Isencephale der Mittelwerte, ist der Ausdruck der Grösse r. Die Streuung der Einzelwerte, d. h. die Entfernung der Einzelwerte von der Isencephalen, ist beim Menschen viel beträchtlicher als bei der Gruppe der Anthro- 
pomorphen und der der niederen Affen (vgl. Fig. 1). Die Cerebralisation ist bei $\mathrm{ihm}$ also viel grösseren Schwankungen unterworfen, die psychischen Differenzen sind viel grösser. Auch innerhalb der Species Mensch lässt sich der schon oben erwähnte Ausschlag des L̇uropäers gegenüber dem Australier auf dieser Figur im grösseren streubild des Europäers erkennen.

Die homogene Gruppe der 20 Australier liegt von allen Individuen am tiefsten, nur wenige Individuen übersteigen die Mittelwertslinie nach oben. Der Cerebralkoeffizient schwankt bei ihnerl zwischen 189 und 247, er ist im Mittel 222. Die psychische Komponente bewegt sich zwischen 704 und 997 mit dem Miltelwert 851. In all dem tritt der intellektuelle Tielsland der Gruppe klar zutage. Dadurch, dass die Gruppe über Individuen von sehr kleinem und sehr grossem Knochenvolumen verfügt, zeigt sie alle Verhältnisse besonders klar. Sie ist eine lllustration für die Tatsache, dass das Cehirn des Australiers auf einer tieferen Entwickelungsstufe stehen geblieben ist als das des Durchschnittsmenschen, eine Tatsache, die durch anatomische Untersuchungen an Australiergehirnen auch erwiesen ist fBolk (29), Duckworth (30), Flashman (31), Karphus (32), Buschan (33)].

Die Europäergruppe nimmt eine wesentlich andere Lage ein. Sic hat in ihren Einzelwerten eine grössere Streuung nach oben und unten, sie ist ja auch nicht so homogen und unter dem Einfluss der Kultur schwankt die Ausbildung des Gehirns beträchtlich. Sie bält sich ganz auf der rechten Seite der Figur, denn der Europäer ist im Vergleich zum Australier plumper, er hat einen viel massigeren Knochenbau, also ein grösseres $V$. Der Miitelwert für den 'Cerebralkoeffizienten der Europäergruppe steht hoch über dem der Australier, fast so hoch über der Durchschnittsisencephalen, wie der Australier darunter. Es kommt dem Europäer im Punkte seiner psychi- 
schen Entwickelung also eine wesentlich höhere Stelle zu, als dem Australier. Der Cerebralkoeffizient schwankt zwischen 217 und 291 und ist in Mittel 253, die psychische Komponente bewegt sich zwischen 805 und 1247 mil dem Mittel 1027.

Die Negritogruppe (6 Individuen) ist zu klein, als dass sich daraus giltige Mittelwerte berechnen lassen. Gleichwohl ist ersichtlich, dass zwei ihrer Individuen eine besonders hohe Stellung in ihrer Schädelkapazität einnehmen. Sie fallen, wie auch der Sandwich-Insulaner aus der Reihe und beeinflussen den Mittelwert sehr stark. Ich enthalte mich jeder Deutung dieser Eigentümlịchkeit, vielleicht waren die einzelnen Skeletteile nicht von demselben Individuum. Die Negritos würden sich in ihren Mittelwerten etwa wie die Europäer stellen, streicht man die beiden fraglichen Skelete, dann stehen sie wesentlich tiefer. Der Cerebralkoeffizient für die übrigbleibenden 4 lndividuen schwankt dann zwischen 238 und 258 und ist im Mittelwert 249,3. Die mittlere psychische Komponente ist. dann 981.

Eine Reihe von Individuen anderer menschlicher Gruppen finden sich auf dem ganzen Streubild des Menschen verteilt. Eine Zusammenfassung aller Mittelwerte gibt die Tabelle II.

Man hat sich in der Literatur besonders viel mit relativen Hirngewichten beschäftigt, als man nachwies, dass das kleinere Tier des relativ grösseren Gehirns bedürfe. Das lässt sich an der relativen. Schädelkapazität natürlich ebensogut erkennen. Die Fig. 4 gibt für den Menschen auf der Ordinate die relativen, das heisst die in Prozenten des Knochenvolumens ausgedrückten Schädelkapazitäten und auf der Abszisse die Knochenvolumina wieder. Da zeigt sich, dass die kleineren Individuen die grössere relative Kapazität besitzen, und dass die relativen Kapazitäten mit zunehmendem Knochenvolumen, das heisst mit zunehmender Massigkeit und Grösse der Indi- 
Tabelle II.

\begin{tabular}{|c|c|c|c|c|}
\hline & $\begin{array}{c}\text { Individuen- } \\
\text { zahl }\end{array}$ & $\begin{array}{c}\text { Mittlere } \\
\text { Kapazitat }\end{array}$ & $\begin{array}{l}\text { Mittleres } \\
\text { Volumen }\end{array}$ & $\begin{array}{c}\text { Mittlere } \\
\text { psychische } \\
\text { Komponente }\end{array}$ \\
\hline Australier. & 20 & 1146 & 742 & 851 \\
\hline Europller. & 11 & 1457 & 1102 & 1027 \\
\hline Negrito & 6 & 1308 & 591 & 1072 \\
\hline Negrito ohne Nr. 34 & & & & \\
\hline und 37. & 4 & 1210 & 563 & 988 \\
\hline Rest. . . . . . & 9 & - & - & - \\
\hline Mensch & 46 & 1276 & 828 & - \\
\hline Anthropomorphen & 12 & 474 & 985 & - \\
\hline Niedere Affen & 14 & 127 & 160 & - \\
\hline
\end{tabular}

viduen nicht kontinuierlich, sondern in einer Kurve fallen. Die Gleichung für diese Kurve lässt sich berechnen. Sie ist, da

$$
\begin{aligned}
\mathrm{K} & =\mathrm{c} \cdot \mathrm{V}^{\mathrm{r}} \\
\mathrm{RK} & =\frac{\mathrm{K} \cdot 100}{\mathrm{~V}}
\end{aligned}
$$

und die Grössen $c=240$ (im Mittel) und $r=0,25$ (im Mittel) bekannt sind:

$$
\mathrm{RK}=\frac{100 \cdot \mathrm{c} \cdot \mathrm{V}^{\mathrm{r}}}{\mathrm{V}}=\frac{24000 \cdot \mathrm{V}^{0,25}}{\mathrm{~V}}
$$

oder, wenn ich statt RK (Relative Kapazität) $=\mathrm{y}$ und statt $\mathrm{V}=\mathrm{x}$ setze :

$$
y=24000 \cdot x^{-0,75}
$$

Fig. 4 gibt die nach dieser Formel konstruierte Kurve wieder. Sie ist ein klarer Ausdruck für die Gesetzmässigkeit, die in den in der Figur eingezeichneten Einzelwerten liegt, und sie legt sich der lang gestreckten Australiergruppe sehr gut an.

Die innerhalb der einzelnen menschlichen Gruppen gewonnenen Resultate lassen sich, da die Grösse $r$ innerhalb der 
Der Gehirnreichtum der Australier und anderer Hominiden etc. 605

Mittelwerte.

\begin{tabular}{|c|c|c|c|c|c|c|}
\hline $\begin{array}{c}\text { Grösse } \\
r \\
(=\operatorname{tg} \alpha)\end{array}$ & $\begin{array}{l}\text { Winkel a } \\
\text { (Neigung } \\
\text { der Isen. } \\
\text { cephalen) }\end{array}$ & $\begin{array}{l}\text { Mittlerer } \\
\text { Cerebral- } \\
\text { koeffi- } \\
\text { zient c }\end{array}$ & $\sigma_{e}$ & $\begin{array}{l}\text { Varia- } \\
\text { tions: } \\
\text { koeff. } \\
\text { zient }\end{array}$ & $\begin{array}{l}\text { Wahrsch. } \\
\text { fehler } \\
\text { von c }\end{array}$ & $\begin{array}{c}\text { Typen- } \\
\text { differenz } \\
\text { der Cerebral- } \\
\text { koeffizienten }\end{array}$ \\
\hline 0,253 & - & 222 & 14,1 & 6,35 & 2,18 & $D$ \\
\hline$\ldots$ & $-\cdots$ & 253 & 24,5 & 9,69 & 4,98 & 346,5 \\
\hline- & - & 265 & 28,4 & 10,72 & 7,82 & \\
\hline- & 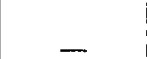 & 249 & 8,2 & 3,29 & 277 & 65,1 \\
\hline- & - & - & - & - & - & $\ldots$ \\
\hline 0,25 & $14^{0}$ & 240 & 26,6 & 11,11 & 2,65 & $\ldots$ \\
\hline 0,43 & $23^{\circ} 20^{\prime}$ & 24,5 & $\ldots$ & - & - & - \\
\hline 0,60 & $31^{\circ}$ & 6,0 & - & - & - & - \\
\hline
\end{tabular}

ganzen Species Mensch als annähernd konstant anzunehmen ist, wie wir gesehen haben, gut miteinander vergleichen, da sie nur durch die verschiedene Grösse des uns vor allem interessierenden psychischen Faktors c, des Cerebralkoeffizienten sich unterscheiden.

Immerhin bedarf hierfür das von Mollis on beim Skeletmaterial eingeführte Gesetz beim Menschen einer Korrektur. Es bringt die Schädelkapazität in Beziehung zum Knochenvolumen und setzt damit voraus, dass das Knochenvolumen seinerseits wieder der Ausdruck eines bestimmten nervös versorgten somatischen Apparates sei. Je grösser das Knochenvolumen, desto grösser sei auch dieser Apparat, Muskeln, Gelenke, Haut usw., desto grössier sei auch ihr nervöses Centrum im Gehirn. Das ist richtig innerhalb der Affenreihe, das stimmt auch noch beim Australier, das stimmt bei anderen wildlebenden Volksstämmen, aber es stimmt nicht mehr beim Kulturinenschen. Alle primitiv lebenden Volksstämme haben einen grazilen Knochenbau, der Kulturmensch aber hat einen wesentlich plumperen. Das ist nach Fischer (34) eine Folge der 
Domestikation. Das Plumperwerden der einzelnen Skeletteile erhöht selbstverständlich das Knochenvolumen beim Europäer um ein beträchtliches, ohne dass damit, wie es die Voraussetzung der Formel ist, auch der am Knochen ansetzende nervös zu versorgende Lokomotionsapparat usw. entsprechend dem

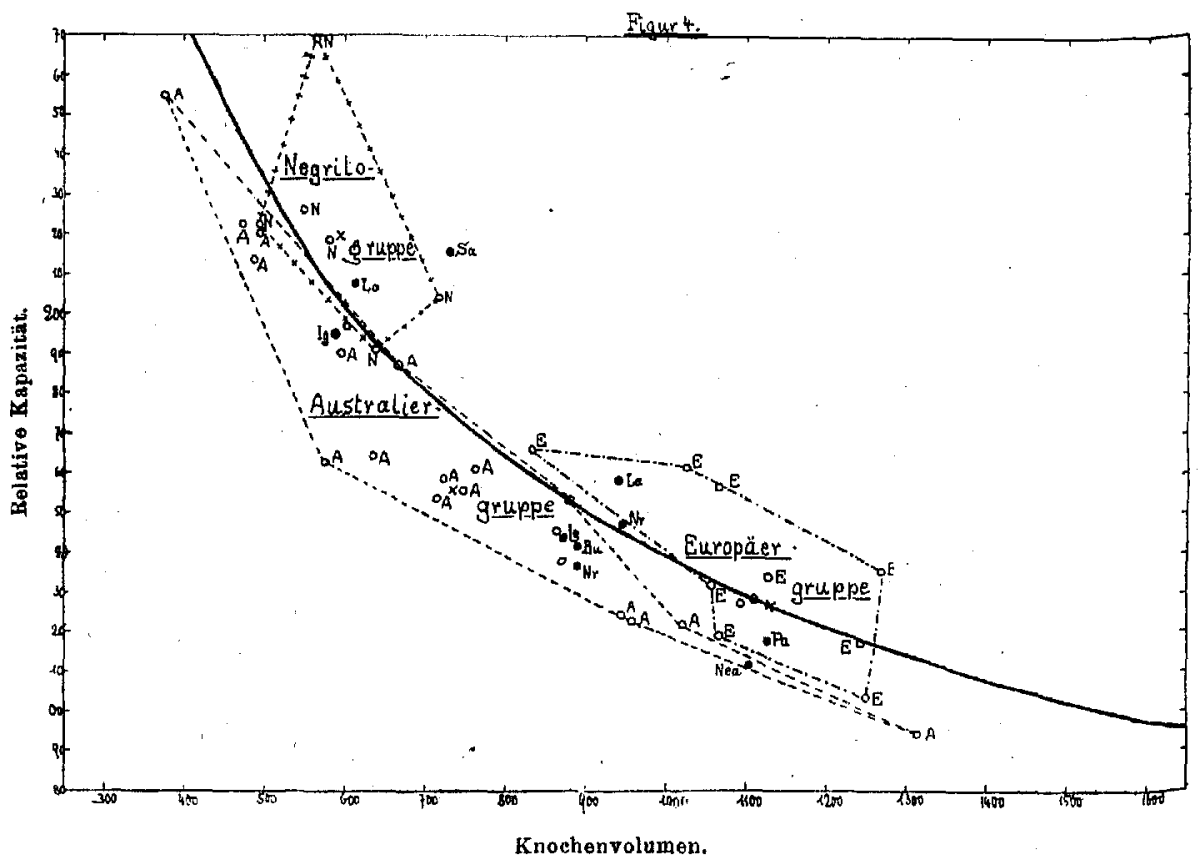

Relative Kapazität und Volumender Extremitätenknochen. Horizontal liegt der Massstab für das Volumen der Extremilätenknochen, vertikal der Mussstab fú die relative (d. h. die in Plozenten des Knochenvolumens ausgedıłukte) Kapazität.

A Australier, E Europäer, $\mathrm{N}$ Negrito. Übrige Bezeichnungen siehe Fig. 3.

Knochenvolumen zunimml. Das erhöhte Knochenvolumen beim Kulturmenschen ist eben einfach nur der Ausdruck einer infolge kultureller Eiriflüsse erhöhten Massigkeit, sonst sagt es nichts.

Das ist beim Europäer in Rechnung zu setzen. Trotzdem eigentlich, wenn man so sagen darf, sein grosses Knochen. 
volumen eine viel $\mathbf{z u}$ grosse somatische Gehirnkomponente für sich in Anspruch nimmt, stellt er sich doch noch psychisch hoch über den Australier, bei dem beides in normalem Verhältnis steht. Aber gegenüber anderen Gruppen, so gegenüber dem Negrito, steht er nicht mehr so hoch da, denn da drückt ihn schon merklich sein $z u$ grosses Knochenvolumen.

Ich habe versucht, diesen Faktor, der das Ergebnis beim Europäer beeinflusst, in Rechnung zu setzen. Bei sämtlichen Individuen, die nir erreichbar waren, wurde an den volumetrisch verwendeten Knochen auch die Länge gemessen. Ich habe mich bei dieser Längenmessung der von Mollison in seiner Arbeit über die Körperproportionen der Primaten (35, S. 84f.) benützten Messverfahren bedient.

Die gefundenen Werte für Längen und Volumina der Einzelknochen sind in der Tabelle III niedergelegt. Es kam nun darauf an, für das wechselseitige Verhältnis beider Grössen einen Ausdruck zu finden, das heisst, einen Index aufzustellen, der die Grösse der Massigkeit der verschiedenen Knochen angab. Um in der gleichen Dimension zu bleiben, wurde das Volumen in Beziehung gesetzt zur dritten Potenz der Länge, und um Zahlen mit vielen Dezimalen zu vermeiden, wurden die erhaltenen Werte mit $10^{5}$ multipliziert. Das Volumen wurde in Kubikzentimeter, die Länge in Zentimeter in Rechnung gesetzt. Die gefundene Verhältniszahl möchte ich Massen index nennen; sie isl gegeben durch die Gleichung:

$$
1=\frac{V \cdot 10^{5}}{L^{3}}
$$

Der Massenindex eines Knochens ist demmach gleich dem Volumen des Knochens, multipliziert mit 100000 und dividiert durch den Kubus der Länge. Die Indices für die Einzelknochen wie auch die Mittelwerte sind aus der Tabelle ersichtlich.

Dieser neu aufgestellte Index ist ein viel richtigerer und klarerer Ausdruck für die Massigkeit, eines Knochens als 
die Indices, die bisher in der Literatur zu diesem Zwecke angegeben wurden. Denn diese stützen sich nur auf lineare Masse, sie setzen Umfang der Diaphysen und Länge, Durchmesser der Diaphysen und Länge zueinander in Beziehung (Martin, 22, S. 928) und geben damit wohl ungefähre Anhaltspunkte, aber keine präzise Grösse für die mehr oder minder starke Plumpheit eines Knochens. Sie sind Behelfskriterien, da man vor Volummessungen der Knochen sich gescheut hat. Das lag zum grossen Teil daran, dass bisher für die Technik dieser Messungen nur schlecht gangbare Wege vorhanden waren.

Auf die Einzelergebnisse der Tabelle III einzugehen, ist hier nicht der Ort, so mag dieses Nebenresultat für sich allein sprechen.

Uns interessiert die Frage: Wie müssen wir dem Europäer die grössere Plumpheit seines Skelets in Rechnung setzen? Wir fassen zu diesem Zwecke nach Berechnung der Mittelwerte für die einzelnen Gruppen die Gesamtvolumina sämtlicher 6 Knochen und ebenso auch die 6 Längen in Summen zusammen und berechnen für diese Summen die entsprechenden Indices. Wir erhalten:

\begin{tabular}{|c|c|c|c|c|}
\hline & $\begin{array}{l}\text { Individuen- } \\
\text { zahl }\end{array}$ & $\begin{array}{l}\text { Gesamt- } \\
\text { volumen } \\
\text { in } \mathrm{ccm}\end{array}$ & $\begin{array}{l}\text { Summe der } \\
6 \text { Längen } \\
\text { in } \mathrm{mm}\end{array}$ & $\begin{array}{l}\text { Massen- } \\
\text { index }\end{array}$ \\
\hline 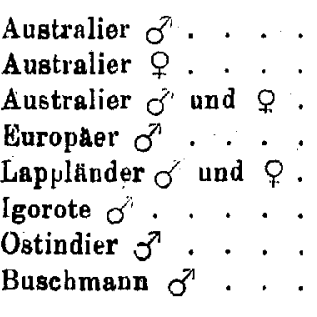 & $\begin{array}{r}9 \\
11 \\
20 \\
10 \\
1 \\
2 \\
1 \\
1\end{array}$ & $\begin{array}{r}915 \\
605 \\
739 \\
1129 \\
617 \\
726 \\
873 \\
791\end{array}$ & $\begin{array}{l}2051 \\
1854 \\
1942 \\
1995 \\
1677 \\
1844 \\
1803 \\
2007\end{array}$ & $\begin{array}{r}10,60 \\
9,49 \\
10,08 \\
14,22 \\
13,07 \\
11,49 \\
14,90 \\
9,78\end{array}$ \\
\hline
\end{tabular}


Der männliche Australier ist also um $\frac{10,60}{14,22}=0,747 \mathrm{in}$ seinem Knochenbau graziler als der Europäer. Unter der Annahme, dass die grössere Massigkeit des Europäerskelets nur durch die Kultur und nicht durch stïrkere Entwickelung der Muskulatur usw. bedingt sei, müssten wir mit dieser Zahl das Knochenvolumen des Europäers multiplizieren, um eine Volumengrösse für seine Knochen zu erhalten, die seine durch die Domestikation erworbene Plumpheit ausschaltet und ihm die Grazilitait wiedergibt, die er im wildlebenden Zustande vielleicht einmal besessen hat, und die vielleicht ein besserer Ausdruck für die Grösse seines innervationsbedürftigen Lokomotionsapparates wäre, als die unkorrigierten Werte.

Der mittleren Kapazität des Europäers $=1457 \mathrm{ccm}$ entspräche dann nicht das Knochenvolumen von $1102 \mathrm{ccm}$, sondern eines von $823 \mathrm{ccm}$. Mit anderen Worten, die eingezeichneten Werte für die Europäer wären auf der Fig. 3 nach links horizontal zu verschieben. Sie würden sich dadurch noch höher über die mittlere Isencephale erheben als sie so schon liegen, und stehen nun erst einigermassen richtig dem Australier und Negrito gegenüber. Ihr psychisches Höherrücken kommt auch zum Ausdruck im Cerebralkoeffizienten, denn dieser wäre dann für den Europäer statt 253 im Mittel 262. Diese Korrektur ist. natürlich nur eine ungeführe, aber immerhin ist sie bei Untersuchungen an Kulturvölkern nicht ausser acht zu lassen.

Wenn man sich einmal nach der Molli s o n schen Methode eir Bild für die Cerebralisation innerhalb der Primaten geschaffen hat, wie es die Fig. 1 veranschaulicht, und wenn man auch bei den Hominiden über genügende Vergleichswerte verfügt, wozu diese Arbeit ein Beitrag sein soll, dann gelingt es leicht, fossile Reste, über deren psychische Qualitäten man sich ein Urteil verschaffen will, in diesen Rahmen einzuordnen. Voraussetzung ist, dass man annähernd ihre Kapazität und 
annähernd das Knochenvolumen ihrer grossen Extremitätenknochen kennt.

Der Neandertaler mit einer Kapazität, die $\mathrm{Schwalbe}$ auf $1230 \mathrm{ccm}$ angibt, und einem Volumen seiner 6 Extremitätenknochen, das Mollis on auf 1106 berechnet hat, steht, wie die Fig. 3 erkennen lässt, hinsichtlich seines Gehimreichtums noch etwas unter dem Durchschnitt der Australier mit grösslem Knochenvoulmen. Sein Cerebralkoeffizient von 212 und seine psychische Komponente von 797 werden, wie die Fig. 2 zeigt, nur von wenigen Australiern an Niedrigkeit übertroffen.

Den Pithecanlhropus erectus, ein viel umstrittenes Objekt, hat schon Mollison (35, S. 394) auf Grund seiner Untersuchungen zwischen die menschliche und die Anthropomorphengruppe stellen können. Der Pithecanthropus hat nach D u bo is eine Kapazilät von 900 bis $1000 \mathrm{ccm}$, sein Femur hat nach Mollis on ein Volumen von ungefähr $646 \mathrm{ccm}$. Es sei nun einerseits die Annahme gemacht, der Pithecanthropus gehöre zu den Anthropomorphen. Sein Knochenvolumen ist, nach Mol. lison, nach dem Mittel der Hylobatiden berechnet, dann $1610 \mathrm{ccm}$. Nach der innerhalb der Anthropomorphen giültigen Formel

$$
c=\frac{K}{V^{0,43}}
$$

berechnet, ist sein Cerebralkoeffizient dann 37,6, während er hei den Anthropomorphen im Mittel' 24,5 ist und 28,1 nicht übersteigt. Berechne ich mit diesem ungünstigen Anthropomorphen-r, um den Menschen ohne Einschränkung vergleichsweise heranziehen zu können, auch den Mittelwert für c beim Menschen, so erhalte ich 71. Der Pithecanthropus steht mit seinem Cerebralkoeffizienten also zwischen beiden Gruppen, er gehört weder der einen noch der anderen an.

Es sei jetzt andererseits angenommen, der Pithecanthropus gehöre zur Gattung Mensch. Dann entspricht, nach dem Mittel- 
wert für Australier berechnet, seinem Femurvolumen ein Gesamtvolumen sämtlicher 6 Extremitätenknochen von $1046 \mathrm{ccm}$. Nach der für den Menschen giltigen Formel

$$
c=\frac{K}{V^{0.26}}
$$

berechnet, hat er dann einen Cerebralkoeffizienten von 158,2 , wïhrend das menschliche Mittel bei 240 liegt und das Anthro pomorphenmittel mit diesem für sie zu günstigen Menschen-r berechnet bei 84,6 liegt.

Während der Pithecanthropus also das eine Mal, wenn ich ihn als Anthropomorphen behandle, sich hoch über das Mittel der Anthropomorphen hinausstellt, bleibt er das andere Mal, wenn ich annahmsweise ihn zum Menschen stempele, tief unter allen Werten der menschlichen Gruppe. Die Wahrheit liegt in der Mitte, er ist weder ein Mensch noch ein Anthropomorphe,

Zusammenfassend komme ich zu dem Ergebnis:

Das von Snell erkannte und von Dubois und La. p i q u e an reichem Material wissenschaftlich präzisierte Geset $z_{\text {, }}$ dass sich die Gehirngewichte bei den Wirbeltieren verhalten wie die 0,58. Potenzen ihrer Körpergewichte, lässt sich am Skeletmaterial nachprüfen.

Es behält seine Giltigkeit für die Primaten niederer Ordnung.

Bei den Anthropomorphen verhalten sich die Kapaziläten bzw. die Gehirngewichte wie die 0,43., beim Menschen wie die 0,25. Potenzen der Knochenvolumina bzw. Körpergewichte.

Diese neu sich ergebende Gesetzmässigkeit findet ihre Er. klärung darin, dass die Gehirnmasse mit zunehmender Körpermasse nicht in all ihren Teilen in gleicher Weise zunimmt, sondern dass der der somatischen Innervation dienende Hirnanteil stark, der für die höheren psychischen Fähigkeiten vorhandene Hirnanteil nur wenig mit zunehmender Körpermasse wächst. 
Die Cerebralisation, das heisst, die höhere Vergeistigung des Gehirns, lässt sich aus der Art dieser Zunahme erkennen. Sie findet ihren zahlenmässigen Ausdruck im Cerebralkoeffizienten der Snellschen Formel und in der psychischen Komponente Mollisons.

Das nach diesen Gesichtspunkten untersuchte Material lässt klar die grosse geistige Kluft zwischen niederen Affen und Anthropomorphen und die noch grössere zwischen Anthropomorphen und Menschen erkennen (Fig. 1).

Innerhalb der Species Mensch hat sich ergeben, dass den beiden untersuchten Gruppen, dem Australier und dem Europäer, eine verschiedene Cerebralisation als Ausdruck ihrer geistigen Fähigkeiten zukommt, dass dem Australier für seine über das rein Körperliche hinausgehenden psychischen Bedürfnisse weniger Gehirn zur Verfügung steht als dem Europäer (Fig. 3).

Es besteht die Möglichkeit, aus dem Skelet eines Individuums einen Schluss auf seine Cerebralisation, d. h. letzten Endes auf die Grösse jenes Hirnanteils zu machen, der die Psyche vermittelt.

Abgeschlossen den 27. Oktoher 1919. 


\section{Literatur.}

1. Brandt, A., Sur le rapport du poids du cerveau à celui du corps chez différents animaux. B. de la soc. Impér. des naturalistes de Moscou 1867. T. 40 . 2. p. $525-543$.

2. Bisch off, Th. L. W. v., Das Hirngewicht des Menschen. Bonn 1880.

3. Retzius, G., Über das Hirngewicht der Schweden. Biol. Unters., N. F. IX. 1900 .

4. Marchand, F., Über das Hirngewicht des Menschen. Abhandl. d. ä̆chs. Gesellsch. d. Wissensch., Math.phys. Klasse, 1902. S. 392—482.

5. Matiegka, H., Uber das Hirngewicht, die Schadelkapazităt und die Kopfform, sowie deren Beziehungen zur psychischen Tatigkeit des Menschen. Sitzungsber. d. böhm. Gesellsch. d. Wissensch., Math.-naturw. Klasse, 1902. XX.

6. Dräseke, G., Gehirngewicht und Intelligenz. Arch. Rassen. und Gesellschaftsbiologie, Bd. 3, S. 499-522.

7. Handmann, E., Uber das Hirngewicht des Menschen auf Grund von 1414 im Pathologischen Institut zu Leipzig vorgenommenen Hirnwagungen. Arch. f. A nat. u. Physiol, 1906. Anat. Abt. S. 1-40.

8. Wendt, W. W., Alte und neue Gehirnprobleme. München 1909.

9. Manouvrier, L, Sur la valeur de la taille et du poids de corps comme terme de comparaison entre la masse de l'encéphale et la masse du corps. Bulletins de la Societé d'Anthropologie. Paris 1882.

10. Derselbe, Sur l'interprétation de la quantité dans l'encéphale et dans le cervea en particulier. Mémoires de la Soc d'Anthrop. Paris. 2. Série. T. 3 (1885). p. $137-326$.

11. Snell, Otto, Die Abhängigkeit des Hirngewichts von dem Körpergewicht und den geistigen Fähigkeiten. Arch. f. Psych, Bd: 22. 1891.

12. Dubois, Eugen, Les poids somatiques et encéphales. Bull, de la Soc. d'Anthrop. Paris 1897. p. 374.

13. Derselbe, Ǔber die Abhängigkeit des Hirngewichts von der Körpergrösse bei den Säugetieren und beim Menschen. Arch. f. Anthrop. Bd. 25. Heft 1 und 2. 1898. S. $1-28$. 
14. Dubois, Eugen, Sur la relation du poids du cerveau au poids du corps chez l'homme. Bull. de la Soc. d'Anthrop. 1897. p. 337.

15. Derselbe, Die gesetzmässige Beziehung von Gehirnmasse zur Kärpergrösse bei den Wirbeltieren. Zeitschr. f. Anthrop. u. Morphol. Bd, 18. Festschr. f. Schwalbe 1914.

16. Lapique, Louis, Tablean général des poids somalique et encéphale dans les espèces animales. Bull. de la Soc. d'Anthrop. Paris 1907. p. 249-270.

17. Derselbe, Les poids encéphalique en fonction du poids corporel entre individus d'une même espèce. Bull. déla Soc. d'Anthrop. Paris 1907. p. 313-345.

18. Derselbe, Comparaison du poids encéphalique entre les deux sexes de l'espèce humaine. Bull. de la Soc. d'Anthrop. Paris 1907. p. 313.

19. Derselbe, Sur la relation da poide de.l'encéphale au poids du corps chez le chien. Soc. de Biol. 1898.

20. Girard, P., Les poids somatique ot encéphalo chez les oiseaux. C. R. de la Soc. de Biol. 1905. p. 665.

21. Eyerich, G. und L. Loewenfeld, Uber die Beziehungen des Kopfumfange zur Körperlünge und zur geistigen Entwickelung. Ref. in Arch. f. Anthrop. N. F. Bd. 4. S. 203.

22. Martin, R., Lebrbuch der Anthropologie. Jena 1914.

23. Molli s o n, T h., Zur Beurteilung des Gehirnreichtums der Primaten nach ihrem Skelet. Arch. f. Anthrop. N. F. XIII. S. 388.

24. Welcker, H., Die Kupazität und die drei Bauptdurchmesser der Schadelkapsel bei den verschiedenen Nationen. 1886. Arch. f. Anthrop. Bd, 16. S. 1.

25. Manouvrier, De la quantité dans l'encépbale. Mémoires de la Soc. d'Anthropol. de Paris 1885.

26. Leuret, Anatomie comparée du système nerveux. Paris 1839-57. I. p. 384. cf. Snell.

27. Kla atsch, Ergebnisse meiner australischen Reise. Korrespondenzbl. d. deutsch. Gesellsch. f. Anthropol, Ethnol. u. Urgeschichte. Jahrg. 38. Nr. 9/12. S. 79-94.

28. Flochrig, P., Gehirn und Seole. 2. Ausg. Lejpzig 1896.

29. Bolk, Das Gehirn eines Papua von Neuguiner. Petrus Campor, Deel III. S. 347-366.

30. Duckworth, W. L. H., The brains of aboriginal natives of Australia in the Anatomy School, Cambridge University. Journ. Anat. and Physiol. Vol. 42. p. 176 u. 271. Ref.: Jahresber. d. Literatur uber phys. Anthrop. p. 830.

31. Flaghman, D. Fr., The morphologie of the brain of the Australian aboriginal. Reports from the Pathol. Laboratory of the Lunacy Depart- 
ment, New South Wales Gouvernement 1908. Vol. 1. p. 3. p. 1-42. Ref.: Zentralbl. f. Anthrop. 1909. p. 279.

32. Karphus, J. P., Utber ein Australiergehirn, nebst Bomerkungen ther einige Negergehirne. Arbeit. a. d. Neurol. Inst. Wien 1902. Heft 9. Ref.: Zentralbl. f. Anthrop. 1904. S. 303.

33. Buschan, G., Kultur und Gehirn. Arch. f. Rassen- u. Gesellschaftsbiologie. 1904. Jahrg. 1. S. 689-701.

34. Fi s cher, Eugen, Die Rassenmerkmale des Menschen als Domestikationserscheinungen. Zeitschr. f. Morph. u. Anthrop. Bd. 18. Stuttgart 1914.

35. Mollison, Th., Die Körperproportionen der Primaten. Morph. Jahrb. Bd. 42. Hoft 1/2. 1910. 
Ta.

$\mathrm{V}=$ Volumen in $\mathrm{ccm} . \mathrm{L}=$ Länge

\begin{tabular}{|c|c|c|c|c|c|c|c|c|c|c|}
\hline & & & & , & & $\mathrm{me}$ & & & adi & \\
\hline & & & z & $\begin{array}{r}03 \\
\\
0\end{array}$ & V. & L. & I. & V. & L. & I. \\
\hline Australier . & . & . 1006 & 2 & $\sigma^{\prime}$ & 142 & 323 & 421 & 43 & 257 & 253 \\
\hline & & 08 & 4 & $\sigma^{x}$ & 156 & 308 & 534 & -- & - & - \\
\hline & & 09 & 5 & $0^{7}$ & -- & $=-$ & - & 66 & 280 & 302 \\
\hline & & 10 & 6 & 9 & 153 & 330 & 426 & 41 & 265 & 221 \\
\hline & & 20 & 9 & 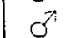 & 93 & 309 & 315 & 29 & 227 & 248 \\
\hline & & 33 & 13 & $0^{1}$ & 95 & 296 & 366 & 29 & 230 & 238 \\
\hline & & 34 & 14 & 万. & 170 & 358 & 370 & 49 & 269 & 251 \\
\hline & & 42 & 16 & $\sigma^{n}$ & 150 & 326 & 433 & 41 & 237 & 308 \\
\hline & & 70 & 19 & $\sigma^{2}$ & 141 & 335 & 422 & 43 & 266 & 228 \\
\hline Australier $\sigma^{7}$ & - & Mittelwert & & & 138 & 323 & 411 & 43 & 254 & 256 \\
\hline Australier . & & . . 1004 & 1 & $q$ & 82 & 297 & 314 & 25 & 227 & 214 \\
\hline & & 07 & 3 & $q$ & 90 & 336 & 238 & 36 & 258 & 209 \\
\hline & & 16 & 7 & q & 87 & 275 & 419 & 30 & 214 & 312 \\
\hline & & 17 & 8 & Q & 112 & 306 & 391 & 33 & 236 & 244 \\
\hline & & 21 & 10 & $q$ & 54 & 266 & 288 & - & - & - \\
\hline & & 28 & 11 & q & 61 & 278 & 284 & 21 & 217 & 206 \\
\hline & & 31 & 12 & q & 68 & 291 & 276 & -- & - & - \\
\hline & & 39 & 15 & ㅇ & 99 & 288 & 416 & 29 & 206 & 833 \\
\hline & & 43 & 17 & $q$ & 92 & 293 & 367 & 33 & 222 & 314 \\
\hline & & 44 & 18 & q & 107 & 291 & 435 & 30 & 225 & 263 \\
\hline & & 73 & 20 & $q$ & 95 & 307 & 328 & 26 & 285 & 202 \\
\hline Australier $Q$ & . & Mittelwert & & & 86 & 294 & 341 & 29 & 227 & 255 \\
\hline Australier $\sigma^{\pi}$ & a. $Q$ & Mittelwert & & & 108 & 306 & 371 & 35 & 239 & 255 \\
\hline Europher . & & 122 & 21 & 0 & 174 & 330 & 485 & 54 & 243 & 378 \\
\hline & & 128 & 22 & $\sigma^{2}$ & 176 & 328 & 499 & 49 & 232 & 392 \\
\hline & & 364 & 23 & $\sigma^{\prime}$ & 153 & 314 & 495 & 46 & 232 & 368 \\
\hline & & 367 & 24 & $\sigma^{7}$ & 168 & 325 & 490 & 51 & 240 & 369 \\
\hline & & 368 & 25 & 0 & 159 & 314 & 513 & 43 & 238 & 318 \\
\hline & & 369 & 26 & 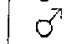 & 158 & 328 & 448 & 46 & 242 & 326 \\
\hline & & 370 & 27 & $\sigma^{7}$ & 153 & 351 & 353 & 47 & 254 & 290 \\
\hline & & 696 & 28 & $0^{-1}$ & 163 & 381 & 450 & 43 & 244 & 296 \\
\hline & & 697 & 29 & $0^{\pi}$ & 179 & 344 & 440 & 53 & 249 & 844 \\
\hline & & 698 & 30 & $\sigma$ & 175 & 320 & 535 & 52 & 253 & 321 \\
\hline Europäer & . & Mittelwert & & & 166 & 329 & 471 & 48 & 243 & 340 \\
\hline Lappländer. & . & 123 & 38 & q & 101 & 287 & 427 & 28 & 199 & 355 \\
\hline & & 124 & 39 & $\sigma^{7}$ & 152 & 294 & 602 & 40 & 212 & 421 \\
\hline Igorote & - & 125 & 40 & $\sigma^{7}$ & 75 & 267 & 396 & 27 & 213 & 277 \\
\hline & & 127 & 41 & 8 & 132 & 300 & 490 & 37 & 249 & 241 \\
\hline Osti & • & 23 & 42 & 0 & 150 & 284 & 654 & 38 & 220 & 358 \\
\hline Buschmann & 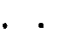 & 46 & 43 & $\sigma^{x}$ & 127 & 310 & 426 & 38 & 243 & 258 \\
\hline
\end{tabular}


Der Gehirnreichtum der Australier und anderer Hominiden etc. 617

bolle III.

in $\mathrm{mm}$. $\mathrm{I}=$ Massenindex.

\begin{tabular}{|c|c|c|c|c|c|c|c|c|c|c|c|}
\hline \multicolumn{3}{|c|}{$U \ln a$} & \multicolumn{3}{|c|}{ Femur } & \multicolumn{3}{|c|}{ Tibia } & \multicolumn{3}{|c|}{ Fibula } \\
\hline v. & L. & I. & V. & L. & I. & v. & L. & I. & V. & L. & I. \\
\hline 39 & 273 & 241 & 414 & 450 & 454 & - & - & - & 46 & 390 & 78 \\
\hline 52 & 259 & 301 & 364 & $4: 3$ & 478 & 214 & $3 \notin 0$ & 459 & 41 & 354 & 94 \\
\hline 72 & 296 & 276 & 526 & 459 & 550 & 474 & 441 & 551 & 67 & 419 & 92 \\
\hline 49 & 284 & 214 & 372 & 449 & 413 & 214 & 401 & 331 & 38 & 401 & 59 \\
\hline 33 & 245 & 226 & 253 & 412 & 360 & 166 & 354 & 375 & 31 & 348 & 74 \\
\hline 34 & 245 & 233 & 306 & 405 & 463 & 224 & 368 & 450 & 34 & 359 & 74 \\
\hline 56 & 283 & 246 & 434 & 468 & 421 & 260 & 408 & 383 & 51 & 400 & 79 \\
\hline 55 & 252 & 338 & 352 & 423 & 464 & 230 & 369 & 461 & 52 & 364 & 108 \\
\hline 44 & 273 & 217 & 432 & 455 & 460 & 286 & 398 & 455 & 45 & 391 & 76 \\
\hline 48 & 268 & 256 & 384 & 438 & 451 & 258 & 387 & 433 & 45 & 381 & 82 \\
\hline 29 & 242 & 204 & 240 & 407 & 359 & 194 & 339 & 216 & 27 & 385 & 72 \\
\hline 41 & 276 & 195 & 342 & 453 & 368 & 212 & 396 & 342 & 39 & 391 & 66 \\
\hline 34 & 235 & 263 & 245 & 375 & 467 & 165 & 338 & 465 & 34 & 318 & 106 \\
\hline 42 & 275 & 202 & 314 & 424 & 412 & 204 & 386 & 355 & 40 & 371 & 83 \\
\hline 20 & 216 & 199 & 150 & 367 & 301 & 119 & 314 & 379 & 19 & 302 & 59 \\
\hline 19 & 231 & 154 & 214 & 379 & 393 & 138 & 329 & 390 & 23 & 320 & 70 \\
\hline 30 & 237 & 226 & 213 & 383 & 380 & 140 & 341 & 354 & 19 & 329 & 54 \\
\hline 33 & 223 & 297 & 223 & 394 & 365 & 160 & 342 & 406 & $\cdots$ & - & - \\
\hline 34 & 240 & 246 & 291 & 411 & 420 & 179 & 347 & 427 & 35 & 338 & 92 \\
\hline 37 & 237 & 278 & 306 & 412 & 437 & 194 & 365 & 400 & 40 & 358 & 80 \\
\hline 31 & 248 & 202 & 277 & 411 & 399 & 182 & 348 & 437 & 23 & 316 & 56 \\
\hline 32 & 242 & 224 & 256 & 401 & 391 & 173 & 349 & 379 & 30 & 341 & 74 \\
\hline 39 & 254 & 238 & $31: 3$ & 418 & 418 & 207 & 365 & 403 & 37 & 360 & 77 \\
\hline 56 & 260 & 320 & 534 & 436 & 643 & 381 & 378 & 706 & 68 & 371 & 136 \\
\hline 51 & 248 & 336 & 486 & 421 & 648 & 261 & $3: 13$ & 707 & 39 & 340 & 99 \\
\hline 49 & 253 & 302 & 518 & 422 & 691 & 302 & 358 & 658 & 57 & 364 & 119 \\
\hline 53 & 260 & 303 & 543 & 426 & 702 & 357 & 355 & 802 & 69 & 354 & 157 \\
\hline 52 & 268 & 291 & 454 & 416 & 630 & 270 & 348 & 643 & 44 & 351 & 101 \\
\hline 58 & 271 & 291 & 486 & 427 & 620 & 291 & 368 & 589 & 50 & 368 & 100 \\
\hline 52 & 273 & 256 & 510 & 463 & 515 & 311 & 402 & 478 & 35 & 397 & 62 \\
\hline 53 & 265 & 291 & 454 & 431 & 568 & 286 & 357 & 629 & 59 & 362 & 124 \\
\hline 59 & 269 & 302 & 589 & 441 & 685 & - & - & - & 55 & $3 \& 1$ & 100 \\
\hline 60 & 285 & 258 & 478 & 423 & 627 & - & - & - & - & - & - \\
\hline 54 & 265 & 295 & 505 & 431 & 633 & 307 & 362 & 653 & 53 & 365 & 111 \\
\hline 32 & 221 & 288 & 270 & 364 & 562 & 153 & 302 & 557 & 33 & 301 & 121 \\
\hline 48 & 234 & 375 & 415 & 387 & 457 & - & - & - & - & -- & - \\
\hline 28 & 235 & 217 & 255 & 367 & 520 & 173 & 333 & 467 & 32 & 328 & 91 \\
\hline 44 & 272 & 211 & 385 & 405 & $5 \& 3$ & 219 & 356 & 487 & 51 & 350 & 118 \\
\hline 42 & 242 & 295 & 364 & 392 & 607 & 250 & 338 & 649 & 49 & 327 & 140 \\
\hline 41) & 262 & 222 & 391 & 436 & 472 & 250 & 380 & 457 & 45 & 376 & 85 \\
\hline
\end{tabular}

